ARTICLE

Received 12 Jan 2015 | Accepted 8 Sep 2016 | Published 25 Oct $2016 \quad$ DOl: 10.1038/ncomms13157

\title{
Replication intermediates that escape Dna2 activity are processed by Holliday junction resolvase Yen1
}

Gizem Ölmezer ${ }^{1,2}$, Maryna Levikova ${ }^{3, \star}$, Dominique Klein ${ }^{1, \star}$, Benoît Falquet ${ }^{1,2}$, Gabriele Alessandro Fontana?, Petr Cejka ${ }^{3} \&$ Ulrich Rass ${ }^{1}$

Cells have evolved mechanisms to protect, restart and repair perturbed replication forks, allowing full genome duplication, even under replication stress. Interrogating the interplay between nuclease-helicase Dna2 and Holliday junction $(H J)$ resolvase Yen1, we find the Dna2 helicase activity acts parallel to homologous recombination (HR) in promoting DNA replication and chromosome detachment at mitosis after replication fork stalling. Yen1, but not the HJ resolvases SIx1-SIx4 and Mus81-Mms4, safeguards chromosome segregation by removing replication intermediates that escape Dna2. Post-replicative DNA damage checkpoint activation in Dna2 helicase-defective cells causes terminal G2/M arrest by precluding Yen1-dependent repair, whose activation requires progression into anaphase. These findings explain the exquisite replication stress sensitivity of Dna2 helicase-defective cells, and identify a non-canonical role for Yen1 in the processing of replication intermediates that is distinct from $\mathrm{HJ}$ resolution. The involvement of Dna2 helicase activity in completing replication may have implications for DNA2-associated pathologies, including cancer and Seckel syndrome.

\footnotetext{
${ }^{1}$ Friedrich Miescher Institute for Biomedical Research, Maulbeerstrasse 66, Basel CH-4058, Switzerland. ${ }^{2}$ University of Basel, Petersplatz 10, Basel CH-4003, Switzerland. ${ }^{3}$ Institute of Molecular Cancer Research, University of Zurich, Winterthurerstrasse 190, Zurich CH-8057, Switzerland. ${ }^{\star}$ These authors contributed equally to this work. Correspondence and requests for materials should be addressed to U.R. (email: ulrich.rass@fmi.ch).
} 
$\mathrm{D}$ uplication of the genome requires the passage of DNA replication forks along the entire length of every chromosome. If segments of DNA remain unreplicated, physical links between the nascent sister chromatids persist, which can lead to aberrant chromosome segregation ${ }^{1}$. Replication fork collapse, characterized by replisome inactivation and DNA breakage, induces recombinogenic DNA lesions and gross chromosomal instability ${ }^{2}$. Consistently, replication stress, which increases the risk of replication fork stalling, arrest, and collapse, has been recognized as a driver in cancerogenesis ${ }^{3}$. Cells respond to replication stress by activating the $S$ phase checkpoint, which triggers a cascade of downstream events aimed at preserving the replication machinery at troubled replication forks until DNA synthesis can resume ${ }^{4}$. Replication restart also involves fork remodelling, nucleolytic processing of stalled replication intermediates and homologous recombination (HR) reactions $s^{5-7}$. Thus, full genome duplication and proper chromosome segregation is dependent on a complicated network of replication and repair proteins that remains incompletely understood.

A protein implicated in multiple aspects of DNA replication and repair is the conserved nuclease-helicase Dna2. Essential in yeast ${ }^{8}, D N A 2$ is required for embryonic development in mice 9 , and its downregulation leads to chromosomal instability ${ }^{10-14}$. The enzymatic activities of Dna2 reside in a RecB-like nuclease domain ${ }^{15}$ with single-stranded DNA (ssDNA)-specific endonuclease activity ${ }^{16}$, and a C-terminal superfamily 1 (SF1) helicase domain $^{8}$; in yeast, Dna2 has an additional, unstructured $\mathrm{N}$-terminal domain that serves a redundant function in $\mathrm{S}$ phase checkpoint activation ${ }^{17}$.

The nuclease activity of Dna2, in particular, has been linked with a number of molecular pathways. In vitro, Dna2 cuts DNA $5^{\prime}$-flaps bound by replication protein A (RPA), and it has been proposed that this activity might facilitate Okazaki fragment maturation by mediating the removal of occasional long $5^{\prime}$-flaps, which might attract RPA and become refractory to cleavage by Rad27 (FEN1 in human) ${ }^{18,19}$. During DNA double-strand break repair, the Dna2 nuclease degrades the $5^{\prime}$-terminated single strand unwound by the Sgs1 helicase (BLM, Bloom syndrome protein in human), promoting DNA end-resection and HR redundantly with Exo1 (ref. 20). Similarly, the Dna2 nuclease has been implicated in Schizosaccharomyces pombe in the processing of stalled replication fork intermediates through degradation of the regressed DNA branch emanating from reversed replication forks as the newly synthesized DNA strands become displaced and anneal with one another to form a chicken-foot structure $^{21,22}$. An analogous reaction, mediated by the DNA2 nuclease in conjunction with Werner's syndrome helicase WRN, promotes replication restart in human cells ${ }^{23}$, while failure to properly control DNA2-mediated DNA resection at stalled forks leads to excessive DNA degradation and genome instability ${ }^{24,25}$.

The physiological role of the Dna2 helicase activity, as opposed to the nuclease activity, has remained unclear. There is currently no evidence that the helicase activity contributes to the degradation/resection of DNA ends at reversed forks or DNA double-strand breaks. Interestingly, a number of Dna2 mutants affected within the conserved SF1 helicase motifs I-VI confer growth defects accompanied by sensitivity to the DNA alkylating agent methyl methanesulfonate (MMS) ${ }^{14,26}$. This phenotype is not generally shared with mutants affected in the N-terminal domain $^{26}$ or nuclease domain ${ }^{27}$, indicating that Dna2 helicase-specific functions in the repair of DNA damage or in the response to damage-induced replication stress exist.

Intriguingly, a genetic screen ${ }^{28}$ uncovered a synthetic sick interaction, characterized by slow growth, between dna2-2, an allele that encodes a Dna2 variant with a single amino acid change (R1253Q) in the helicase domain ${ }^{14}$, and structure-specific $R A D 2 / X P G$ superfamily nuclease YEN1, indicating a potential functional interplay. Yen1, and its human orthologue GEN1, are Holliday junction $(\mathrm{HJ})$ resolvases $^{29}$. These enzymes are best known for their role in processing late $\mathrm{HR}$ intermediates, such as fully double-stranded DNA (dsDNA) four-way HJ junctions ${ }^{30}$. Eukaryotes use three conserved HJ resolvases, Yen1/GEN1, Mus81-Mms4/human MUS81-EME1 and Slx1-Slx4/human SLX1-FANCP to remove recombination intermediates that form during replication-associated DNA repair processes in mitotic cells ${ }^{31}$. Mounting evidence suggests that Mus81-Mms4/MUS81-EME1 also targets unproductive replication intermediates, effectively breaking stalled replication forks to allow HR-dependent replication restart or repair ${ }^{32}$. In human cells, MUS81-EME1 promotes the expression of chromosomal fragile sites, which is thought to represent controlled breakage of underreplicated DNA at the time of mitosis to limit sister chromatid non-disjunction ${ }^{33,34}$. At present, there is no evidence for a similar role of Yen1/GEN1 in targeting replication-rather than recombination-intermediates.

Here, we analyse the interplay between Dna2 and Yen1 to reveal new aspects of the cellular response to replication stress. We find that the Dna2 helicase activity acts on replication fork stalling, promoting full genome duplication along a pathway parallel to HR-mediated replication fork recovery. If the Dna2 helicase fails to respond properly to stalled replication forks, replication intermediates remain and give rise to post-replicative chromosomal DNA links that preclude chromosome segregation. Resolution is uniquely dependent on the actions of Yen1, which identifies a first non-redundant function of Yen1 in protecting cells from mitotic catastrophe after replication stress.

\section{Results}

$\mathrm{Dna}^{\mathrm{R} 1253 \mathrm{Q}}$ is helicase defective and nuclease proficient. Using budding yeast, Campbell and colleagues have conducted a large-scale genetic screen ${ }^{28}$ using dna2-2 (R1253Q) and nuclease-defective allele dna2-1 (P504S) ${ }^{14}$, which identified 37 synthetic sick/synthetic lethal interactions, predominantly with genes involved in DNA replication and repair. Many interactions were shared between dna2-2 and dna2-1, but a synthetic sick interaction with YEN1 was unique to dna2-2. $\mathrm{Dna} 2^{\mathrm{R} 1253 \mathrm{Q}}$ is affected at an invariant arginine in helicase motif IV of the SF1 helicase domain, suggesting that the Dna2 helicase and Yen1 may function in related pathways. However, since single amino acid changes in Dna2 have been described that impact both the nuclease and ATPase/helicase activities ${ }^{35}$, and because $\mathrm{Dna} 2^{\mathrm{R} 1253 \mathrm{Q}}$ has never been isolated and analysed biochemically, we first assessed directly the mutant protein's ATPase/helicase and nuclease activities. Dna $2{ }^{\mathrm{R} 1253 \mathrm{Q}}$ was purified to near-homogeneity following overexpression in Saccharomyces cerevisiae (Fig. 1a,b), and tested alongside wild-type Dna2, and well-established ${ }^{35}$ nuclease-dead and helicase-dead variants, $\mathrm{Dna} 2^{\mathrm{E} 675 \mathrm{~A}}$ and $\mathrm{Dna} 2^{\mathrm{K} 1080 \mathrm{E}}$, respectively.

When wild-type Dna2 was incubated with 5 -tailed DNA, the activity of the ATPase/helicase domain was readily detected, before the potent Dna2 nuclease degraded the ssDNA tails, so that the ATPase was no longer stimulated and ATP hydrolysis subsided; the nuclease-dead variant $\mathrm{Dna}^{\mathrm{E} 675 \mathrm{~A}}$ exhibited persistent ATPase activity ${ }^{35,36}$ (Fig. 1c). In contrast to wild-type and Dna $2^{\mathrm{E} 675 \mathrm{~A}}$, Dna2 $2^{\mathrm{R} 1253 \mathrm{Q}}$ showed no ATPase activity, and was indistinguishable from previously characterized ${ }^{35,36}$ ATPase/helicase-dead variant Dna2 ${ }^{\mathrm{K} 1080 \mathrm{E}}$ (Fig. $1 \mathrm{c}$ and Supplementary Fig. 1). In vitro, Dna2 exhibits ssDNA-specific nuclease activity on $5^{\prime}$-tailed or $3^{\prime}$-tailed DNA substrates, while RPA stimulates its nuclease and enforces $5^{\prime}-3^{\prime}$ directionality, 

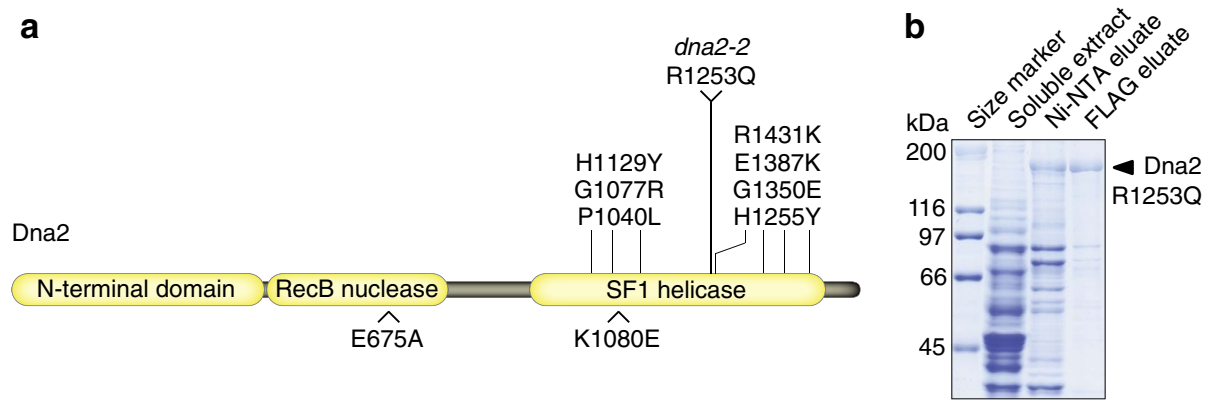
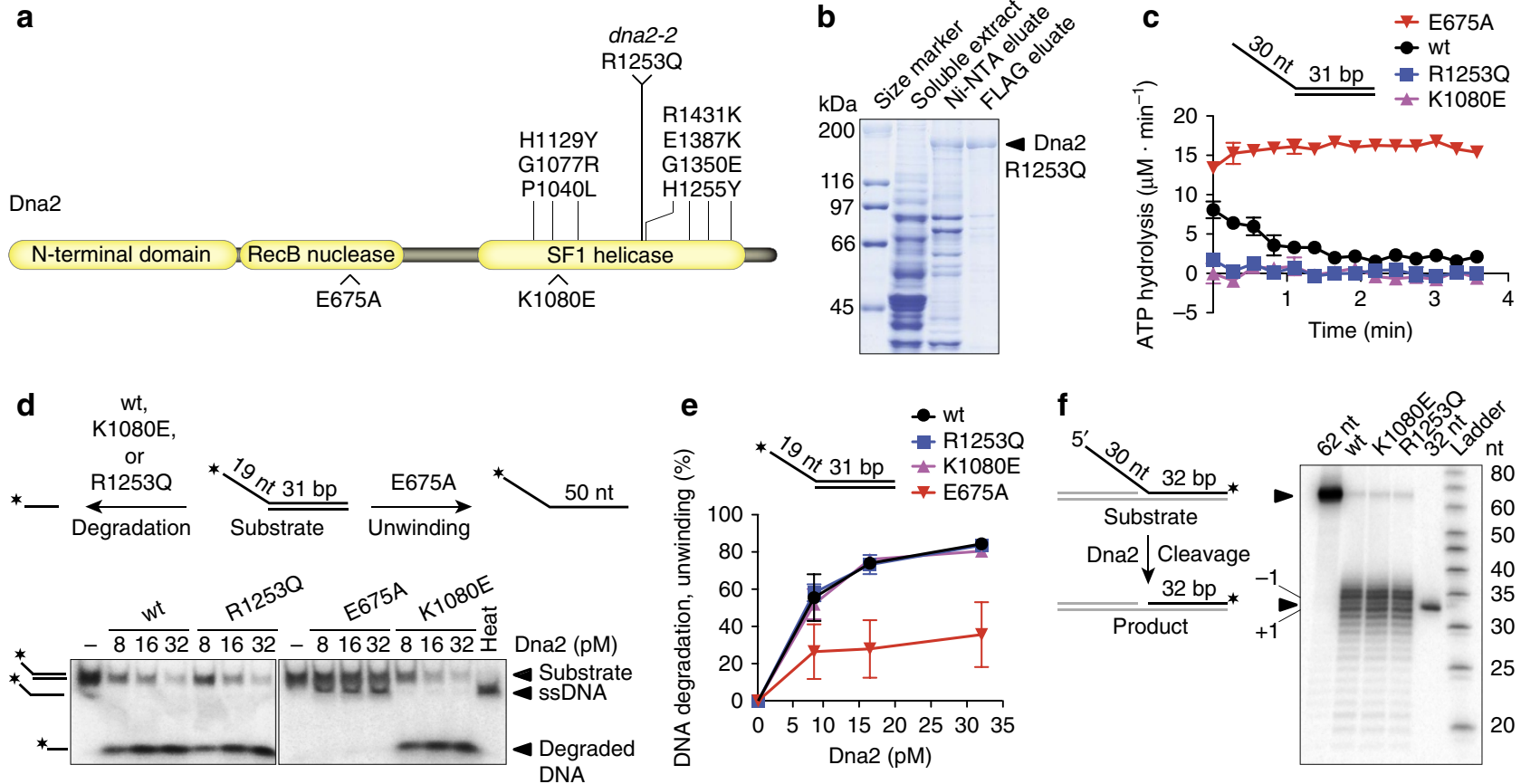

Figure 1 | Biochemical analysis of Dna2 variant R1253Q. (a) Domain structure of Saccharomyces cerevisiae nuclease-helicase Dna2. Above, single amino acid changes within the helicase domain of Dna2 that result in MMS sensitivity, including R1253Q, encoded by dna2-2. Below, position of mutations E675A and K1080E, which have been shown to inactivate the Dna2 nuclease or helicase activity, respectively. (b) Dna2 ${ }^{\text {R } 1253 Q}$ carrying $6 \times$ His and FLAG tags was expressed in S. cerevisiae and purified by affinity chromatography using nickel-nitrilotriacetic acid (Ni-NTA)-agarose and anti-FLAG affinity gel. Fractions were analysed by polyacrylamide gel electrophoresis followed by Coomassie blue staining. (c) Kinetics of ATP hydrolysis by wild-type (wt) Dna2 and indicated variants (all $4 \mathrm{nM}$ ) in the presence of a $5^{\prime}$-tailed DNA substrate $(1 \mu \mathrm{M}$ nucleotides). Data are presented as mean values \pm s.e.m. $(n=2)$.

(d) Processing of $5^{\prime}$-tailed DNA by Dna2. The panel shows a representative $10 \%$ polyacrylamide gel with reaction products after incubation of the DNA substrate with the indicated DNA2 variants and RPA $(16.8 \mathrm{nM})$. ${ }^{*}$, position of the ${ }^{32} \mathrm{P}$-label on the DNA. Heat, heat-denatured DNA substrate.

(e) Quantification of experiments such as those shown in d. Data are presented as mean values \pm s.e.m. $(n=2)$. (f) $5^{\prime}$-flap cleavage by Dna2 variants (all $2 \mathrm{nM})$ in the presence of RPA ( $30 \mathrm{nM})$. Reaction products were separated on a $20 \%$ polyacrylamide denaturing urea gel. Cleavage at the base of the flap produces a radiolabelled fragment of $32 \mathrm{nt}$.

which is likely the relevant polarity in vivo ${ }^{20}$. In the presence of RPA, Dna2 ${ }^{\mathrm{R} 1253 \mathrm{Q}}$ degraded $5^{\prime}$-tailed DNA in a manner similar to wild-type Dna2, showing that the R1253Q mutation does not interfere with the nuclease activity (Fig. 1d). In line with the observed lack of ATPase activity, we did not find evidence of DNA unwinding by Dna $2^{\mathrm{R} 1253 \mathrm{Q}}$, an activity that was readily detected for nuclease-deficient mutant Dna2 ${ }^{\mathrm{E} 675 \mathrm{~A}}$ (Fig. 1d). Quantification of the nuclease/helicase assays showed that Dna2 ${ }^{\mathrm{R} 1253 \mathrm{Q}}$ was as efficient as wild-type and Dna2 helicase mutant K1080E in degrading 5'-tailed DNA (Fig. 1e). Finally, and in accord with previous studies using other Dna2 helicase mutants ${ }^{37,38}$, Dna2 $2^{\mathrm{R} 1253 \mathrm{Q}}$ was fully proficient in removing $5^{\prime}$-flaps from dsDNA by cleavage at the flap base, in a reaction that mimics the potential role of Dna2 in Okazaki fragment processing (Fig. 1f). These results show that the dna2-2 allele confers a helicase-specific defect and does not impinge on the activity of the Dna2 nuclease.

Checkpoint activation and loss of YEN1 impair dna2-2 cells. Having established that the R1253Q mutation selectively inactivates the helicase activity of Dna2, we introduced the dna2-2 allele into cells to investigate the effect of Dna2 helicase deficiency in vivo. While Dna2 protein levels were unaffected (Supplementary Fig. 2a), the R1253Q mutation caused MMS sensitivity, as expected for dna2-2 cells ${ }^{14}$ (Supplementary Fig. 2b). Under unperturbed conditions, the dna2-2 strain exhibited a plating efficiency similar to wild-type. In contrast, viability dropped sharply for the dna2-2 yen $1 \Delta$ double mutant to $\sim 35 \%$ of wild-type levels (Fig. 2a). Doubling time measurements revealed that the dna2-2 mutation was associated with a mild slow growth phenotype, extending doubling times by $\sim 10 \mathrm{~min}$ (103 min versus $92 \mathrm{~min}$ for wild-type). On deletion of YEN1, the growth phenotype was much more severe, with an increase in doubling time of $\sim 50 \mathrm{~min}$ for the double mutant $(143.5 \mathrm{~min}$ ). Consistent with previous results ${ }^{28}$, we did not observe a synthetic growth defect when YEN1 was deleted in Dna2 nuclease-mutant dna2-1 cells (data not shown), indicating that the genetic interaction between YEN1 and DNA2 relates specifically to the Dna2 helicase activity. Contrary to a reported temperature-dependent lethal interaction between YEN1 and DNA2 (ref. 28), we found double mutant cells were viable at elevated temperature $\left(37^{\circ} \mathrm{C}\right.$ ) (Supplementary Fig. $2 \mathrm{c}$ ), although doubling times for dna2-2 and dna2-2 yen1s were further increased by $\sim 20$ and $\sim 5 \mathrm{~min}$, respectively.

Microscopic inspection of exponentially growing dna2-2 cultures revealed an accumulation of cells in G2/M phase of the cell cycle, and this effect was further accentuated on deletion of YEN1. Morphological examination showed that dna2-2 cultures contained $\sim 4 \%$ large dumbbell-shaped cells. In dna2-2 yen $1 \Delta$ cultures, this sub-fraction was more extensive, accounting for $\sim 8 \%$ of cells, and $\sim 5 \%$ of cells exhibited morphological changes such as bud elongation and the formation of short chains of elongated cells (Fig. 2b). Finally, the vast majority of $\mathrm{G} 2 / \mathrm{M}$ cells within the dna2-2 and dna2-2 yen $1 \Delta$ cultures $(\geq 70 \%)$, but not within wild-type or yen1s cultures, contained unsegregated nuclear DNA positioned near the bud neck (Fig. 2b). 

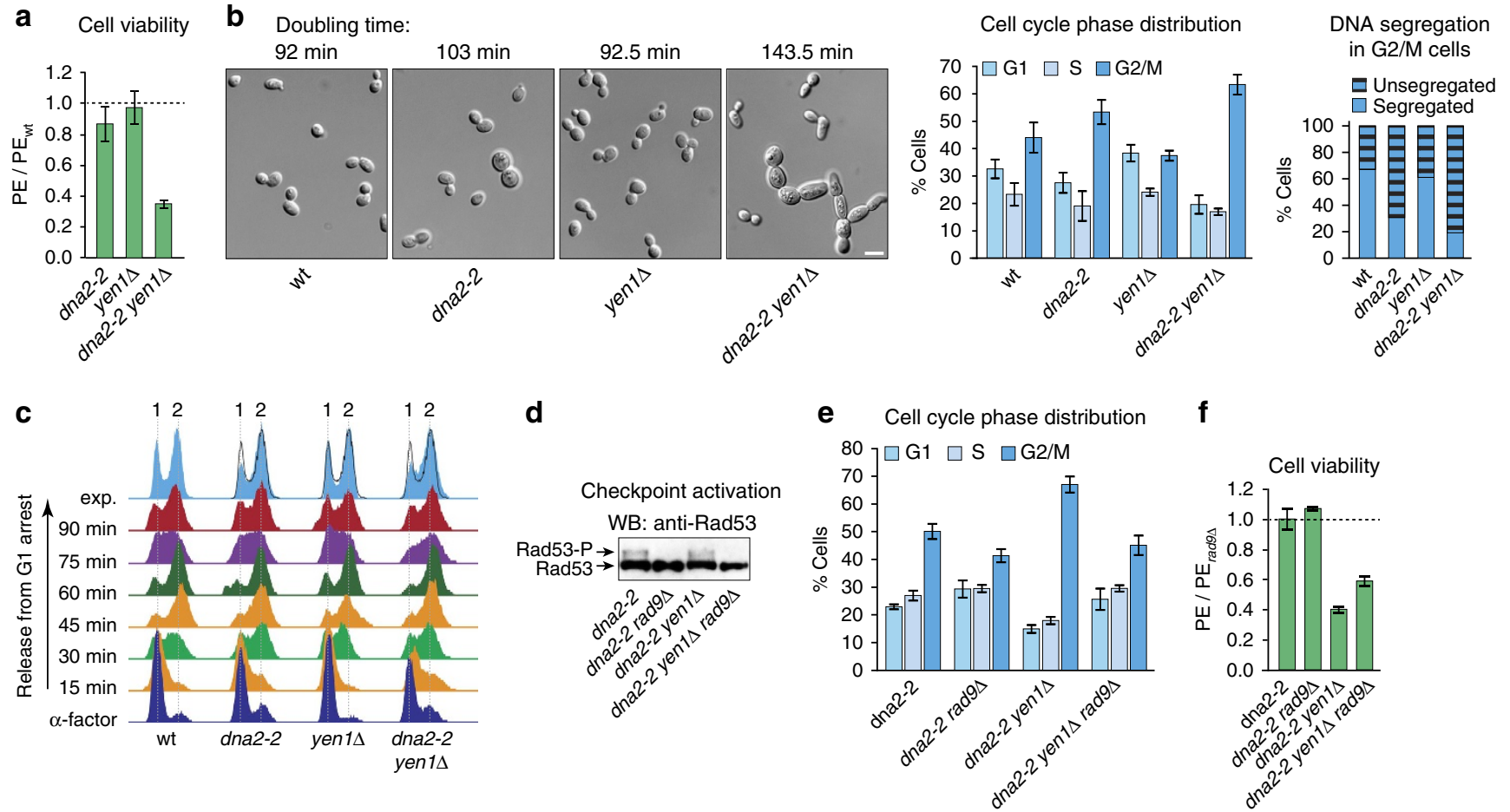

Figure 2 | Dna2 helicase-defective cells suffer dual growth inhibition by checkpoint activation and loss of YEN1. (a) Cell viability of the indicated strains assessed by colony outgrowth. The mean plating efficiency (PE) \pm s.e.m. $(n=3)$ is presented relative to wild-type. (b) Microscopic analysis of the indicated strains growing exponentially in rich medium. Left, representative images (DIC) showing morphological changes associated with Dna2 helicase dysfunction and loss of Yen1. Average doubling times are given $(n=3)$. Scale bar, $5 \mu \mathrm{m}$. Centre, mean distribution of G1, S, and G2/M cells \pm s.e.m. ( $n=4)$. Right, DNA segregation in G2/M cells as determined by DAPI-staining. An average of 200 cells were scored per strain. (c) Flow cytometric analysis of the indicated strains synchronized in G1 using $\alpha$-factor and released into YPAD. The position of cells with 1 and $2 \mathrm{~N}$ DNA content is indicated. Topmost tracks are asynchronous cultures overlaid with the outline of the wild-type profile, showing that Dna2 helicase-defective strains accumulate cells with a $2 \mathrm{~N}$ content over time. (d) Western blot analysis showing chronic low-level DNA damage checkpoint activation in Dna2 helicase-defective cells as indicated by Rad53 phosphorylation (Rad53-P). (e) Effect of DNA damage checkpoint disruption by deletion of RAD9 on the distribution of G1, S, and G2/M cells in exponentially growing cultures of the indicated strains. Data presented as mean distribution \pm s.e.m. $(n=3)$. (f) Cell viability of the indicated strains assessed by colony outgrowth. The mean plating efficiency \pm s.e.m. $(n=3)$ is presented relative to a rad $9 \Delta$ control.

While an accumulation of G2/M cells occurred during exponential growth, analysis by flow cytometry showed that dna2-2 and dna2-2 yen $1 \Delta$ cells progressed through a single cell cycle with apparently normal kinetics on synchronous release into $S$ phase after $\alpha$-factor pheromone-induced G1 arrest (Fig. 2c). This indicates that the helicase activity of Dna2 is largely dispensable for bulk DNA synthesis, but that Dna2 helicase-deficient cells have a tendency to arrest at the G2/M transition, as noted previously ${ }^{14}$. Importantly, replication and the G2/M transition phenotype were unaffected by the presence or absence of Yen1. The accumulation of G2/M cells may result from elevated levels of stochastic DNA damage, since we detected low-level phosphorylation of the checkpoint kinase Rad53 in dna2-2 and dna2-2 yen $1 \Delta$ cells in unperturbed conditions, which was suppressed on deletion of the DNA damage checkpoint mediator RAD9 (Fig. 2d). Moreover, the levels of G2/M cells in either strain were much reduced in the absence of $R A D 9$, and bud-elongation and cell-chain formation was no longer observed (Fig. 2e and data not shown). The extended doubling times for the dna2-2 and dna2-2 yen $1 \Delta$ strains were reduced on RAD9 deletion, albeit not to wild-type levels (96 and $122 \mathrm{~min}$, respectively). Significantly, the viability of both dna2-2 and

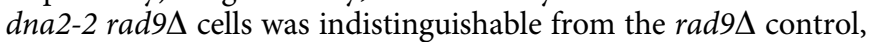
whereas the severe reduction of viability we had observed on loss of YEN1 in the dna2-2 background was only mildly suppressed by $R A D 9$ deletion (Fig. 2f). Together these data suggest that growth defects in dna2-2 yen $1 \Delta$ cells arise from two separate sources: (1)
Dna2 helicase dysfunction causes cells to accumulate DNA lesions during unperturbed growth, triggering Rad9-dependent DNA damage checkpoint activation and a delay at the G2/M transition. (2) Yen1 cannot prevent these defects, so that they manifest themselves similarly in dna2-2 yen $1 \Delta$ double mutant and dna2-2 single mutant cells. Yet, the absence of Yen 1 is toxic to dna2-2 cells, indicating that Yen1 acts downstream, resolving a catastrophic DNA metabolic event that ensues when the Dna2 helicase is non-functional.

Yen1 is critical in $\boldsymbol{d n a 2 - 2}$ cells under replication stress. Dna2 helicase deficiency sensitizes cells to DNA alkylating agent $\mathrm{MMS}^{14,26}$ (Supplementary Fig. 2b). To test whether loss of Yen1 has an additional effect on the MMS sensitivity of Dna2 helicase-defective cells, we exposed dna2-2 yen1s cells to increasing amounts of the drug. As expected ${ }^{39}$, loss of YEN1 alone did not result in overt MMS sensitivity. In contrast, dna2-2 yen $1 \Delta$ cells proved to be several orders of magnitude more sensitive than dna2-2 cells in drop assays (Fig. 3a). This phenotype was not restricted to MMS, and similar results were obtained with topoisomerase I poison camptothecin (CPT) and ribonucleotide reductase inhibitor hydroxyurea (HU). These drugs have disparate mechanisms of action, but their effects (DNA damage, accumulation of trapped Top1 cleavage complexes throughout the genome and nucleotide depletion, respectively) all inhibit the progression of replication forks, 

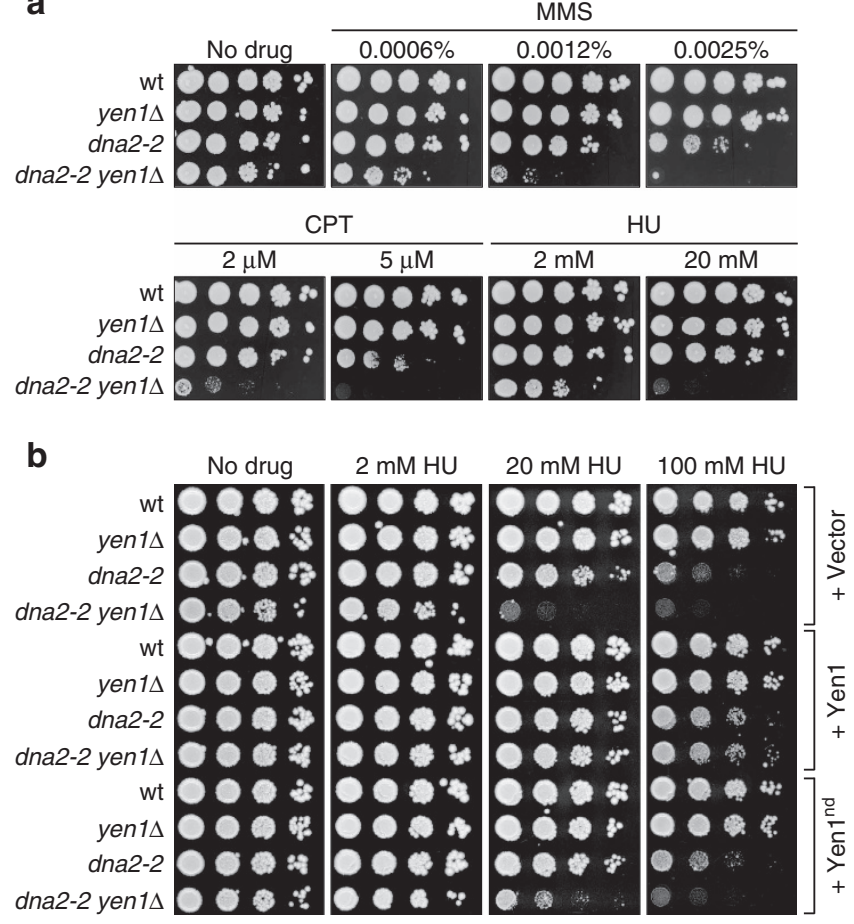

Figure 3 | Dna2 helicase dysfunction and loss of Yen1 nuclease activity synergistically sensitize cells to exogenous replication stress. (a) Drop assays to determine the drug-sensitivity of the indicated strains were done by spotting normalized tenfold serial dilutions of exponentially growing cells onto YPAD plates containing the indicated amounts of MMS, CPT or HU. (b) Analysis of the effects of Yen1 overexpression. Cells of the indicated strains were transformed with empty vector, or derivatives encoding wildtype or nuclease-deficient (Yen $1^{\text {nd }}$ ) versions of Yen1, and plated on YPAD medium in the presence or absence of $\mathrm{HU}$.

suggesting that the functional overlap of Dna2 and Yen1 relates to replication fork stalling. This also suggests that endogenous replication problems are responsible for the growth defects of dna2-2 and dna2-2 yen $1 \Delta$ cells in unperturbed conditions.

Plasmid-based expression of Yen 1 suppressed the HU sensitivity phenotype of dna2-2 yen $1 \Delta$ cells. This suppression was strictly dependent on the nuclease activity of Yen1, demonstrating that Yen1 protects dna2-2 cells through nucleolytic cleavage of otherwise toxic DNA intermediates (Fig. 3b).

DNA damage follows acute replication stress in dna2-2 cells. To investigate the immediate effects of replication stress on Dna2 helicase-defective cells, we next performed mitotic time-course experiments (Fig. 4). Synchronized dna2-2 and dna2-2 yen1s cells were released into $S$ phase under mild replication stress conditions in the presence of $50 \mathrm{mM} \mathrm{HU}$, which impairs, but does not block, replication. After $2 \mathrm{~h}$, cells were shifted back to drug-free medium. DNA synthesis was monitored by flow cytometry, while DNA replication/DNA damage checkpoint activation was assessed by Western blot analysis of the phosphorylation status of Rad53. As expected, wild-type and yen $1 \Delta$ cells exhibited slowed replication progression in the presence of HU. Interestingly, dna2-2 and dna2-2 yen $1 \Delta$ cells progressed through $S$ phase at a pace similar to wild-type. As shown in Fig. 4a, all strains showed S phase checkpoint activation in the presence of $\mathrm{HU}$, and $\mathrm{S}$ phase checkpoint silencing occurred with normal kinetics across strains, followed by completion of bulk DNA synthesis in drug-free medium. One hundred twenty minutes after removal of $\mathrm{HU}$, wild-type and yen $1 \Delta$ cells

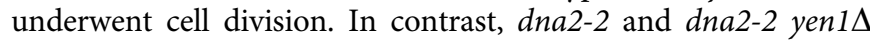
cells exhibited a reemergence of $\operatorname{Rad} 53$ phosphorylation and remained in G2/M with a $2 \mathrm{~N}$ DNA content. This biphasic Rad53 phosphorylation pattern, with an unexpected second wave of checkpoint activation in $\mathrm{G} 2 / \mathrm{M}$ phase, required both the presence of the dna2-2 allele and replication stress in the preceding $S$ phase (that is, it was not discernible above background in control experiments without HU; Supplementary Fig. 3). One interpretation of these observations is that the Dna2 helicase is involved in an immediate response to replication fork stalling, preventing the emergence of DNA structures that signal DNA damage in G2/M.

The presence of Yen1 could not protect Dna2 helicasedefective cells from G2/M checkpoint activation and cell cycle arrest after acute replication stress. However, in the presence of Yen1, the G2/M arrest proved more transient. Thus, G1 cells with a $1 \mathrm{~N}$ DNA content started to appear $240 \mathrm{~min}$ after removal of $\mathrm{HU}$, and continued to appear through overnight incubation in the dna2-2 culture, while the dna2-2 yen $1 \Delta$ strain produced very few G1 cells, as judged by flow cytometry (Fig. 4a). Microscopic analysis showed that within the dna2-2 yen $1 \Delta$ culture $\sim 11 \%$ of cells had segregated their nuclear DNA, while the dna2-2 culture contained a significantly higher number of cells, $\sim 32 \%$, with segregated DNA after overnight incubation (Fig. 4b). This correlated with a significantly lower lethality scored for dna2-2 mutants ( $\sim 28 \%$ viability compared to wild-type) than for the dna2-2 yen $1 \Delta$ double mutant $(<1 \%$ viability compared to wild-type) (Fig. 4c). We conclude that Yen1 promotes mitotic exit with viable chromosome segregation in Dna2 helicase-defective cells recovering from acute replication stress.

Yen1 resolves dna2-2 post-replicative chromosome links. A potential explanation for the apparent slow recovery of dna2-2 cells from G2/M checkpoint arrest relates to recent work showing that Yen1 activity is cell cycle-regulated, with cyclin-dependent kinase (CDK)-mediated phosphorylation lowering its catalytic activity and inhibiting access to the nucleus in S and G2 phase ${ }^{40}$. Upon anaphase onset, Cdc14-dependent dephosphorylation activates Yen1 and allows the protein to accumulate inside the nucleus during mitosis. To test whether Yen 1 cell cycle control is manifest in Dna2 helicase-defective cells recovering from acute replication stress, we expressed and monitored a functional (Supplementary Fig. 4a) version of Yen1, tagged with enhanced green fluorescent protein (EGFP), in dna2-2 and wild-type cells. The expected bi-phasic checkpoint activation of dna2-2 cells in response to acute HU treatment was recapitulated in the presence of Yen1-EGFP (Supplementary Fig. 4b), and the fusion protein exhibited the characteristic cell cycle-dependent subcellular localization reported for Yen1 (Fig. 5a $)^{40}$. Importantly, when cells accumulated as large-budded G2/M cells after HU wash-out, a subset of cells was doublenucleated with a nuclear Yen1-EGFP signal, indicating that mitotic entry had occurred. This subset of cells was markedly larger in case of the wild-type strain after $2 \mathrm{~h}$ in drug-free medium (Fig. 5b). After $4 \mathrm{~h}$ in drug-free medium, the fraction of wild-type G2/M cells diminished as cells underwent mitosis. In contrast, dna2-2 cells remained mostly in G2/M, with Yen1-EGFP in the cytoplasm and a single nucleus at the bud neck, as expected for DNA damage checkpoint-mediated pre-anaphase arrest. Thus, targeting of Yen1 to the nucleus through the actions of Cdc14 (ref. 40) remained largely blocked, showing that unscheduled DNA damage checkpoint signalling in dna2-2 cells is associated with retention of Yen 1 in the cytoplasm, and that this may 


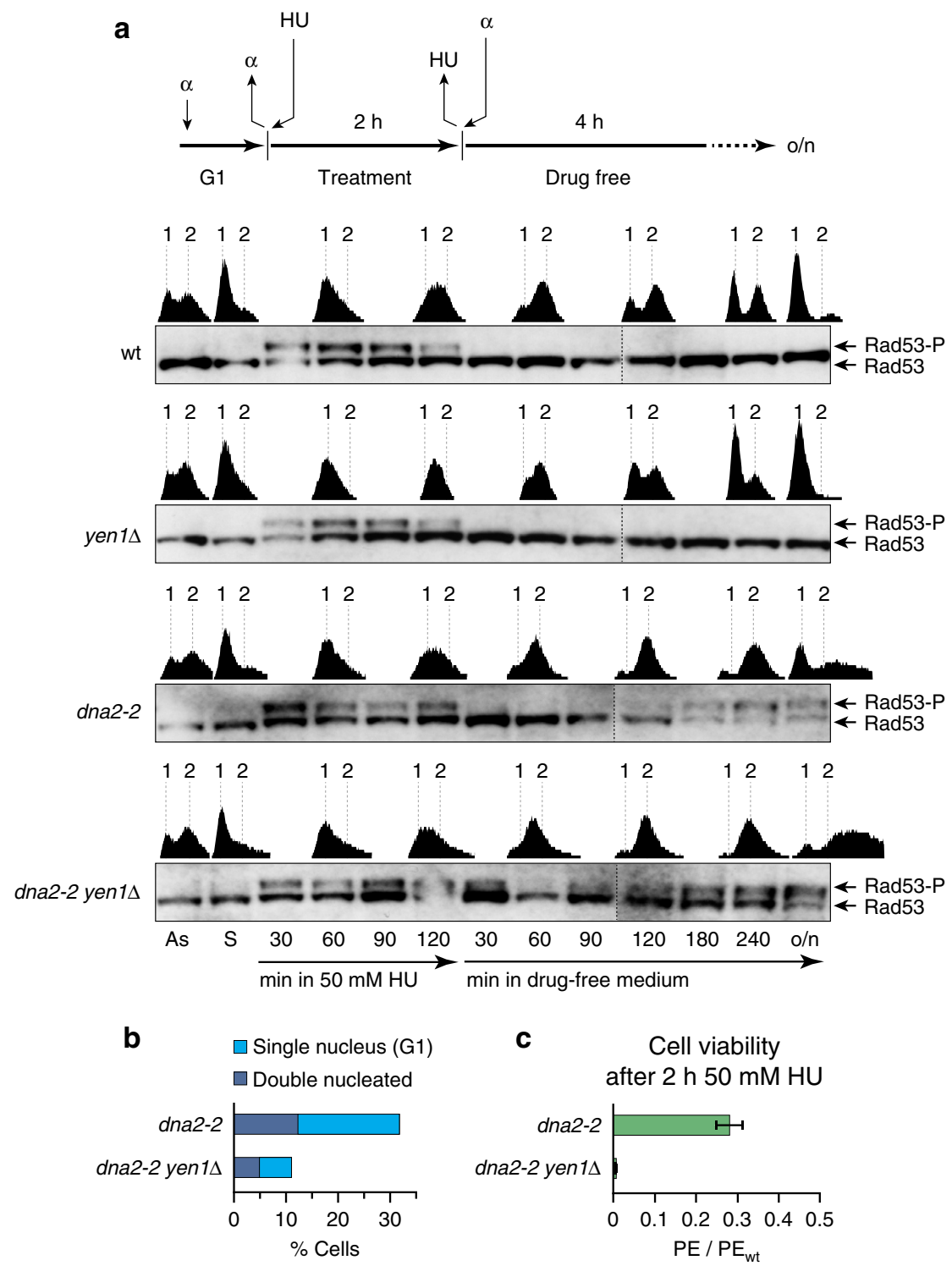

Figure 4 | Dna2 helicase-defective cells are defective in the response to acute replication stress and require Yen1 for subsequent growth. (a) Mitotic time-courses were performed as indicated. Cells, synchronized in G1, were released into acute replication stress in medium containing $50 \mathrm{mM} \mathrm{HU}$ for $2 \mathrm{~h}$, followed by drug wash-out and incubation in drug-free medium with $\alpha$-factor. Checkpoint activation and the progression of DNA replication were monitored by Western blot analysis of Rad53 phosphorylation (Rad53-P) and flow cytometry (1 and 2 N DNA content indicated). As, asynchronous; S, synchronous; $\mathrm{o} / \mathrm{n}$, overnight. (b) Quantification of single-nucleated G1 cells and double-nucleated G2/M cells in the indicated overnight yeast cultures shown in a. Two hundred cells per strain were analysed by microscopic inspection. (c) Viability of the indicated strains after acute replication stress. Cells were plated on drug-free YPAD medium and colony formation was quantified. The mean plating efficiency (PE) \pm s.e.m. $(n=3)$ is presented relative to wild-type.

represent a major impediment to the recovery of Dna2 helicasedefective cells from replication stress.

To test this idea further, we next disrupted the G2/M DNA damage checkpoint by deletion of $R A D 9$, allowing unrestrained anaphase entry, and thus Yen1 activation, in Dna2 helicasedefective cells. Upon acute replication stress treatment, checkpoint activation during $S$ phase occurred normally in the absence of $\operatorname{Rad} 9$, consistent with signalling in response to replication fork stalling, rather than DNA damage, through the intact Mec1-Ddc2/Mrc1/Rad53-dependent pathway. In contrast, unscheduled Rad53 phosphorylation in G2/M phase after $\mathrm{HU}$

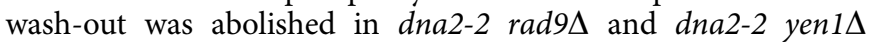
rad $9 \Delta$ cells (Fig. 5 c). Dna2 helicase-defective cells now progressed to cell division with kinetics similar to those exhibited by the rad9s control strain, and we were able to study the effects of Yen1 by microscopic inspection. This revealed two important phenotypes associated with concomitant loss of Dna2 helicase function and Yen1. Sixty minutes after the removal of $\mathrm{HU}$, dna2-2 yen $1 \Delta$ rad9 $\Delta$ samples contained roughly threefold higher levels of early anaphase cells characterized by an elongated nucleus stretched through the bud neck, as compared to dna2-2 rad $9 \Delta$ and rad $9 \Delta$ samples. Concomitantly, there was a delay in the appearance of G1 cells containing a single nucleus (Fig. 5d). This suggests that chromosome segregation and cytokinesis were physically impeded in the absence of Yen1. Consistently, and exclusively in dna2-2 yen $1 \Delta$ rad9 $\Delta$ cells, we observed prominent chromosomal DNA bridges that span the bud neck and connect the segregating masses of nuclear DNA (13.6 and $8 \%$ of the double-nucleated cells affected 180 and $240 \mathrm{~min}$ after HU wash-out, respectively) (Fig. 5e). In some instances this phenotype could be seen in cells approaching abscission, as indicated by a narrowing bud neck. 
a

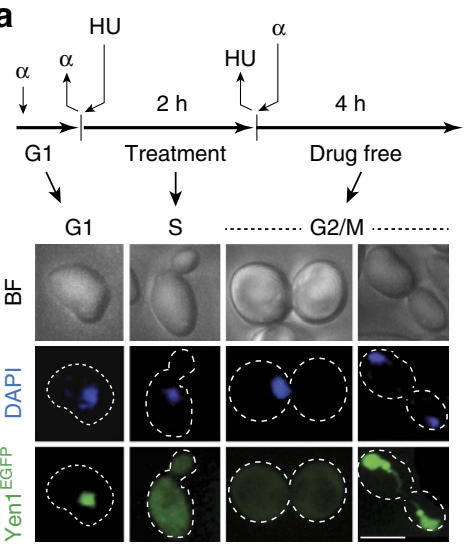

b
$\square$ Nuclear Yen1
$\square$ Cytoplasmic Yen1
$\boxminus$ Nuclear Yen1, double-nucleated

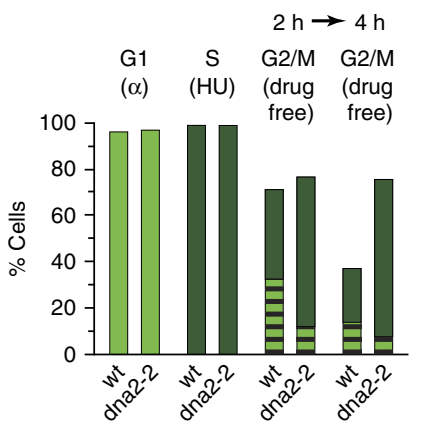

C
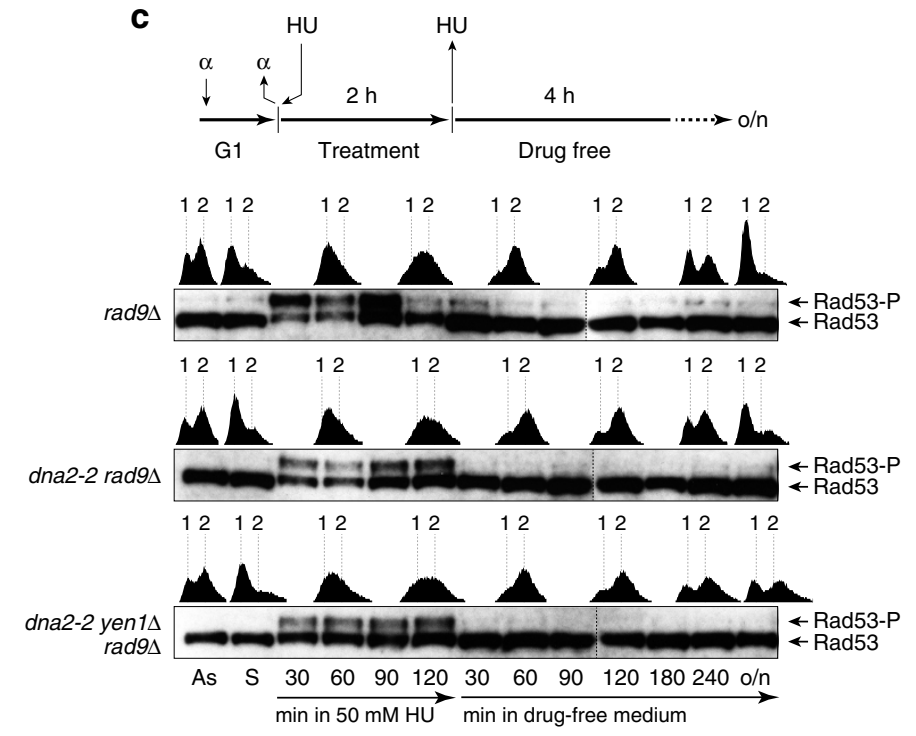

d

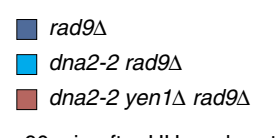

60 min after HU wash-out

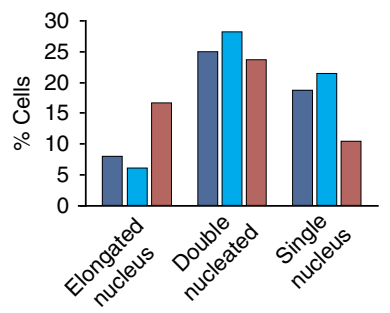

e dna2-2 yen $1 \Delta$ rad9s
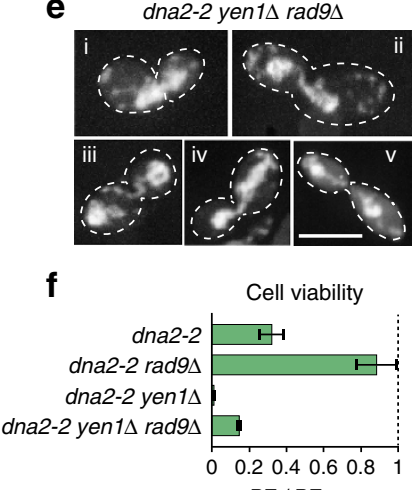

Figure 5 | Post-replicative chromosomal links in Dna2 helicase-defective cells and mitotic resolution by Yen1. (a) Subcellular localization of Yen1 in dna2-2 cells exposed to acute replication stress. Mitotic time-courses were performed as indicated with wild-type and dna2-2 cells expressing Yen1-EGFP. Samples were analysed for nuclear and cytoplasmic localization of Yen1-EGFP following $\alpha$-factor arrest, $1 \mathrm{~h}$ after release into HU-containing medium, and 2 and $4 \mathrm{~h}$ after drug wash-out. Representative images are shown. Scale bar, $5 \mu \mathrm{m}$. (b) Quantitative view of Yen1-EGFP localization as determined in experiments such as those shown in a ( $\geq 100$ cells scored for $\mathrm{G} 1$ and $\mathrm{S}$ phase per strain; $\geq 200$ cells 2 and $4 \mathrm{~h}$ after drug wash-out). (c) Mitotic time-course experiment with DNA damage checkpoint-disrupted strains, performed as indicated and analysed as described for Fig. 4a. (d) Relative distribution of cells in early anaphase (elongated nucleus), late anaphase (double-nucleated) and post-cytokinesis (single-nucleated), as determined by microscopic inspection of samples from c, $60 \mathrm{~min}$ after $\mathrm{HU}$ wash-out ( $\geq 100$ cells scored per strain). (e) Representative microscopic images showing an early anaphase cell with elongated nucleus spanning the bud neck (i), and late anaphase cells with chromosomal DNA bridges between the segregated masses of nuclear DNA (ii-v), a phenotype exclusively observed in dna2-2 yen1 $\Delta$ rad9 $\Delta$ cells. Cells treated as described for $\mathbf{c}$. Scale bar, $5 \mu \mathrm{m}$. (f) Cell viability of the indicated strains, treated as in $\mathbf{c}$, assessed by colony outgrowth. The mean plating efficiency (PE) \pm s.e.m. ( $n=3$ ) is presented relative to rad9 $\Delta$. 
When we determined the effect of checkpoint disruption on cell viability, we found that deletion of RAD9 increased the viability of Dna2 helicase-defective cells after acute replication stress treatment threefold, reaching levels very similar to those observed for the rad $9 \Delta$ control strain. In the absence of YEN1, viability was also improved, but did not reach more than $\sim 14 \%$ of the viability of the rad9 $\Delta$ control (Fig. 5f). Checkpoint activation in Dna2 helicase-defective cells after acute replication stress therefore appears futile, and eliminating the G2/M checkpoint enabled a highly effective Yen1-dependent survival pathway, while allowing a small subset of cells to survive in a Yen1-independent manner. As expected, checkpoint disruption had no beneficial effect when cells were exposed to chronic replication stress (Supplementary Fig. 5), consistent with improved survival being linked specifically to allowing Yen1 access to post-replicative lesions in Dna2 helicase-defective cells after acute replication stress. These observations resonate with previous findings showing that the lethality of some temperature-sensitive dna2 alleles, and of $d n a 2 \Delta$, can be suppressed by deleting RAD9 (refs 14,41,42), linking this phenomenon, at least for the Dna2 helicase-defective dna2-2 allele, to the removal of the inhibitory effect of the G2/M DNA damage checkpoint on Yen1 activation.

To test whether Yen1 activation, not mitotic entry per se, is sufficient for Yen1 to resolve aberrant DNA intermediates that arise in Dna2 helicase-defective cells, we used a constitutively active form of Yen1, referred to as Yen $1^{\text {on }}$ (Supplementary Fig. 6a). Yen $1^{\text {on }}$ is not controlled by CDK and therefore permanently active and nuclear ${ }^{43}$. We expressed Yen $1^{\text {on }}$ from a galactose-inducible promoter in G2/M in dna2-2 yen $1 \Delta$ cells recovering from acute, $\mathrm{HU}$-induced replication stress in the presence of nocodazole. Strikingly, Yen $1^{\text {on }}$-expressing cells did not exhibit unscheduled DNA damage checkpoint activation during nocodazole-induced G2/M arrest (Supplementary Fig. 6b). Furthermore, transient expression of Yen $1^{\text {on }}$ in dna2-2 cells recovering from acute HU treatment led to a significant increase in cell viability $(\sim 3.3$ fold \pm 0.24 s.e.m., $n=2$ ), as determined by colony outgrowth.

Collectively, these results suggest that Dna2 helicase-defective cells fail to respond adequately to replication stress, leading to postreplicative DNA damage signalling and chromosome entanglements. Upon anaphase entry, Yen 1 promotes the survival of Dna2 helicase-defective cells by resolving post-replicative chromosomal DNA links, allowing proper chromosome segregation.

Yen 1 acts distinct from $\mathrm{HJ}$ resolution in dna2-2 cells. Yen1 is known for its role in removing persistent HJ DNA structures that accumulate as Rad52-dependent HR intermediates. Previous findings also suggest an increased requirement for Rad52-dependent DNA repair by HR in Dna2 helicase-defective cells. Thus, dna2-2 rad52 $\Delta$ cells without overt growth defect at $30^{\circ} \mathrm{C}$, but temperature sensitivity at a restrictive temperature of $37^{\circ} \mathrm{C}$, have been described ${ }^{44}$. We generated dna2-2 rad52 $\Delta$ cells and found that compared to $d n a 2-2(103 \mathrm{~min})$ and $\mathrm{rad} 52 \Delta$ (112 $\mathrm{min})$, double mutant cells grew slowly, even at $30^{\circ} \mathrm{C}$, with a doubling time of $144 \mathrm{~min}$. Furthermore, loss of Rad52 led to pronounced synthetic hypersensitivity of dna2-2 cells to HU (Fig. 6a). Finally, we observed a significant increase of spontaneous Rad52 foci, indicative of $\mathrm{HR}^{45}$, in dna2-2 cells in unperturbed conditions (Fig. 6b), whereas Dna2 focus formation is elevated in rad52 $\Delta$ cells $^{46}$. This suggests that Dna2 and Rad52-dependent HR represent parallel and compensatory pathways in the response to replication stress.

Elevated levels of HR repair could explain why loss of Yen1 is detrimental to dna2-2 cells. If so, dna2-2 mus81A cells should exhibit an even stronger growth defect than dna2-2 yen $1 \Delta$ cells, given that Mus81-Mms4 is activated, in a CDK-dependent manner, prior to Yen1 activation at anaphase onset. Reaching an activity peak in its hyperphosphorylated state in $\mathrm{G} 2 / \mathrm{M}$, Mus81-Mms4 is thus the major nuclease in removing $\mathrm{HR}$ intermediates in budding yeast ${ }^{40}$. Notwithstanding, we found that in contrast to loss of Yen1, disruption of Mus81-Mms4, or the Slx1-Slx4 HJ resolvase, did not increase the doubling time of dna2-2 cells in unperturbed conditions. In the presence of $\mathrm{HU}$ or MMS, deletion of SLX1 had no effect on the sensitivity of Dna2 helicase-defective cells. Deletion of MUS81, which in itself results in replication stress sensitivity, added to their sensitivity (Fig. 6c and data not shown), consistent with a requirement for Mus81-Mms4 in the resolution of excessive HR intermediates in dna2-2 cells. However, dna2-2 yen $1 \Delta$ cells were significantly more sensitive to HU or MMS than dna2- 2 mus $81 \Delta$ cells ( 0.2 versus $45 \%$ cell survival at $20 \mathrm{mM} \mathrm{HU}$ as determined by colony outgrowth) (Fig. 6c,d and data not shown), despite the fact that defects related to $\mathrm{HJ}$ resolution as a consequence of Yen1 loss have been shown to transpire only in the absence of a functional Mus81-Mms4 resolvase ${ }^{39}$. Therefore, there is a pathway, distinct from canonical HJ resolution, which uniquely requires Yen1 for the removal of DNA intermediates that are apparently not amenable to cleavage by Mus81-Mms4, in dna2-2 cells. Indeed, the toxicity caused by loss of Yen 1 cannot be explained by an accumulation of HR intermediates alone, as we found that the synthetic sick relationship between DNA2 and YEN1 is maintained in cells deleted for RAD52, which cannot engage in $\mathrm{HR}$ reactions. In fact, we were unable to generate a dna2-2 rad52 $\Delta$ yen $1 \Delta$ triple mutant by tetrad dissection (data not shown), and have confirmed an essential requirement for Yen1 in dna2-2 rad52 $\Delta$ cells using a plasmid-based assay (Fig. 6e). These results contrast with an epistatic relationship that exists between RAD52 and the HJ resolution pathway defined by MUS81-MMS4 and YEN1 (refs 39,47), and imply that the structures that are targeted by Yen 1 in order to maintain the viability of Dna2 helicase-defective cells derive from perturbed replication intermediates in a HR-independent manner.

Yen1 resolves DNA replication intermediates in $d n a 2-2$ cells. To address the question whether replication fork stalling in Dna2 helicase-defective cells gives rise to an accumulation of DNA intermediates that might become targets for Yen1, we turned to the natural replication fork barrier $(\mathrm{RFB})^{48}$ within the ribosomal DNA (rDNA) on chromosome XII. We compared rDNA from actively replicating wild-type and dna2-2 mutant cells by two-dimensional (2D) gel electrophoresis and monitored the disappearance of replication intermediates as cells progressed from $S$ phase to nocodazole-induced G2/M arrest. In $S$ phase, Dna2 helicase-defective cells showed a pattern of replication intermediates very similar to wild-type (Fig. 6f). As expected, replication intermediate levels dropped significantly when cells accumulated in G2/M during nocodazole arrest. However, the resolution of replication intermediates, in particular of RFB-stalled and converged forks, was less efficient in dna2-2 cells, leading to a $\sim 2$ and $\sim 3$-fold less prominent decrease compared to wild-type, respectively. These results are in good agreement with previous observations of accumulating stalled and converged fork intermediates within the rDNA of dna2-2 cells ${ }^{49}$, and corroborate the notion of an aberrant response to replication fork stalling in Dna2 helicase-defective cells. To see if Yen1 targets aberrant replication intermediates that persist in Dna2 helicase-defective cells, we expressed, in asynchronous dna2-2 cultures, constitutively active Yen $1^{\text {on }}$ (Fig. 6g), allowing us to monitor Yen1 actions prior to the activation of endogenous Yen1 and chromosome segregation in M phase. Similar to staged $\mathrm{S}$ phase cells, 2D gel electrophoresis of exponentially growing 
a

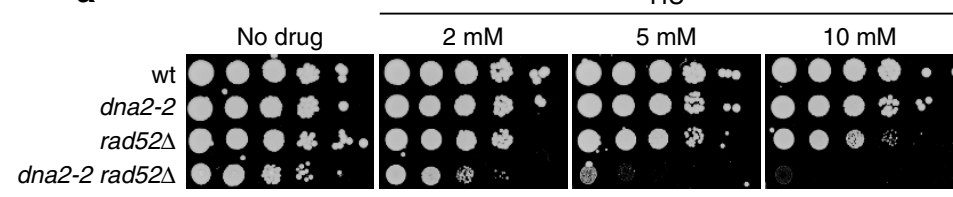

C

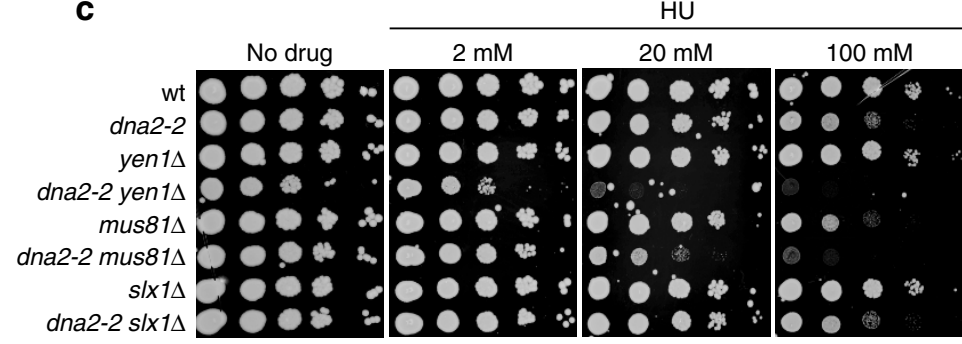

d

b Rad52-YFP foci

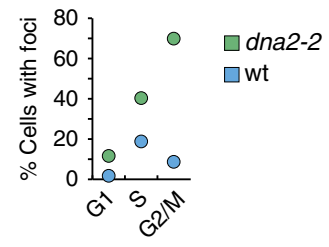

d Cell viability

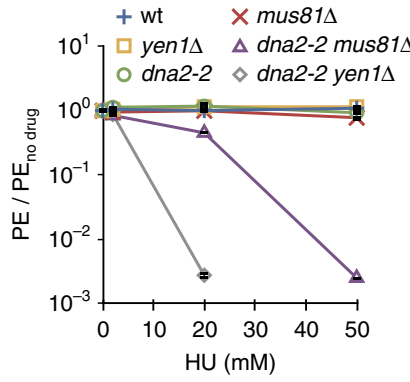

g dna2-2 + empty vector

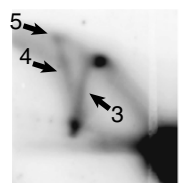
$30 \mathrm{~min}$ $\alpha$-factor exp. 12

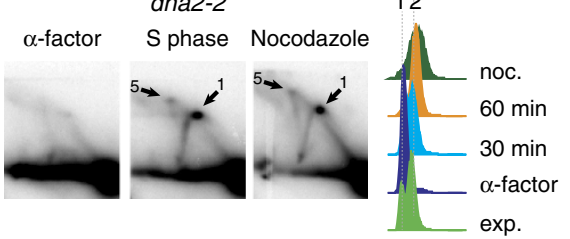

(2) Small-Y

(3) Large- $Y$

(4) X-spike

(5) Converged

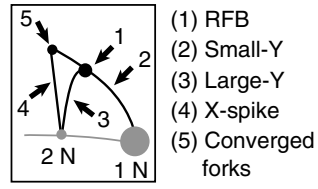

Resolution of DNA intermediates

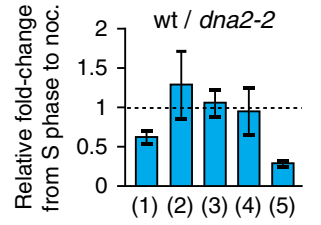

12

e

Cells + pDNA2 (URA3)

Ura-

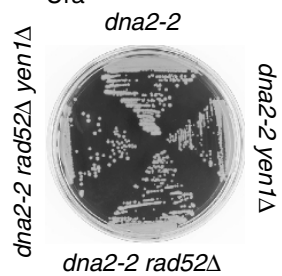

dna2-2 rad52 $\triangle$

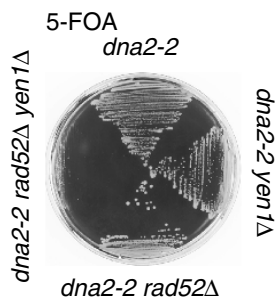

dna2-2

+ Yen $1^{\text {on }}$

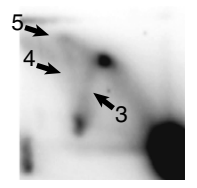

12

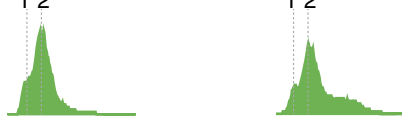

DNA intermediates Yen $1^{\text {on }} /$ empty vector

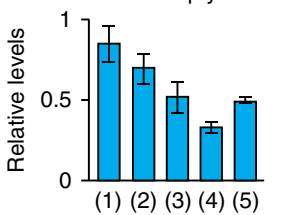

Figure 6 | Yen1 uniquely resolves toxic DNA intermediates in Dna2 helicase-defective cells along a pathway distinct from canonical HJ resolution. (a) Synergistic defects in the resistance to replication stress in homologous recombination-deficient dna2-2 rad52 cells. Drop assay performed as in Fig. 3a. (b) Spontaneous Rad52-YFP foci in wild-type and dna2-2 cells in different cell cycle stages, determined by microscopic analysis ( $\geq 180$ cells scored per strain). (c) Genetic interactions of dna2-2 with HJ resolvases YEN1, MUS81-MMS4 and SLX1-SLX4. Drop assays performed as in Fig. 3a. (d) Mean plating efficiency $(P E) \pm$ s.e.m. $(n=3)$ of the indicated strains assessed by colony outgrowth in the presence of increasing amounts of HU, relative to nodrug conditions. (e) Interaction between dna2-2, RAD52 and YEN1. Cells of the indicated genotypes and containing a plasmid expressing wild-type DNA2 ( $\mathrm{pDNA2}$ ) were grown under uracil selection to ensure retention of the pDNA2, or on medium containing 5-FOA to select against the plasmid. Failure to grow on 5-FOA is indicative of an inviable genotype. (f) Analysis of replication and recombination intermediates in the rDNA of wild-type and dna2-2 cells traversing S phase into nocodazole-induced G2/M arrest. Genomic DNA was digested with Bg/ll and subjected to 2D gel electrophoresis. The fragment probed by Southern hybridization contained the rDNA autonomously replicating sequence (ARS), the 5S transcriptional unit, and the RFB in its centre, as indicated. DNA structures chosen for quantification included RFB-arrested forks (1), Y-arc structures containing a replication fork at varying positions outside the RFB $(2,3)$, the X-spike indicative of four-way branched DNAs containing Holliday junctions or hemicatenanes (4), and forks converging at the RFB (5). Representative autoradiographies are marked for RFB-stalled (1) and converged replication fork intermediates (5), which were resolved less efficiently in dna2-2 cells compared to wild-type following $S$ phase. Three independent experiments were quantified and the data are presented as mean values \pm s.e.m. (g) 2 D analysis as in f, but using exponentially growing dna2-2 cells expressing or not Yen1 ${ }^{\text {on }}$. Intermediates that were reduced upon Yen ${ }^{\text {on }}$ expression are indicated. Three independent experiments were quantified and the data are presented as mean values \pm s.e.m.

dna2-2 cells showed the expected rDNA replication intermediates and, in addition, a more prominent signal indicative of recombination intermediates (X-spike), consistent with an accumulation of $\mathrm{G} 2 / \mathrm{M}$ cells with increased rates of $\mathrm{HR}^{49}$. Yen $1^{\text {on }}$ did not affect the RFB signal, suggesting that replication forks arrested at the barrier are not immediately susceptible to Yen 1 nuclease activity. In contrast, and consistent with the ability of Yen1 to resolve recombination intermediates, moderate constitutive $\mathrm{Yen} 1^{\text {on }}$ expression markedly reduced the $\mathrm{X}$-spike signal. Importantly, single fork intermediates ( $\mathrm{Y}$ structures) and converged forks were also decreased on Yen $1^{\text {on }}$ expression, showing that Yen1, in addition to resolving four-way X-DNA, is able to remove replication intermediates that accumulate in Dna2 helicase-defective cells. These results suggest that endogenous Yen1, activated on anaphase entry, uniquely resolves persistent replication fork/converging fork structures to disentangle 
underreplicated nascent sister chromatids when Dna2 helicasedefective cells enter mitosis, thereby safeguarding chromosome segregation and enabling viable mitotic exit.

\section{Discussion}

Our analyses of the interplay between Dna2, HJ resolvase Yen1 and the DNA damage checkpoint allows us to define important functions of the Dna2 helicase activity and the Yen1 nuclease in the replication stress response. We propose a model, where the Dna2 helicase activity represents a HR-independent replication stress response pathway that helps to ensure full replication of the genome. Replication intermediates that escape the attention of Dna2 persist and impair sister chromatid separation, unless they are resolved by Yen1 in mitosis. Thus, the actions of Yen1, which has so far only been known to target HR intermediates, allow viable chromosome segregation along a novel pathway, distinct from canonical HJ resolution (Fig. 7).

The precise constitution of the DNA structures that threaten chromosome segregation in Dna2 helicase-defective cells remains to be determined. However, the fact that Yen1 can detoxify them indicates that these DNA intermediates conform to the substrate spectrum of Yen1, which includes $5^{\prime}$-flaps and fully double-stranded DNA three-way and four-way junctions ${ }^{29,43}$. As Dna2 ${ }^{\mathrm{R} 1253 \mathrm{Q}}$ retains $5^{\prime}$-flap endonuclease activity (Fig. 1f), this activity of Yen1, which could potentially support the proposed role of Dna2 in Okazaki fragment processing ${ }^{18,19}$, is unlikely to explain the requirement for Yen1 in Dna2 helicase-defective cells. Consistently, we find no genetic indication that Yen1 might support functions of the major Okazaki fragment processing nuclease Rad27 in vivo (Supplementary Fig. 7). We thus favour the possibility that the capacity of Yen1 to target branched dsDNA intermediates is relevant for the protection of dna2-2 cells. This ability distinguishes Yen1, Mus81-Mms4 and Slx1-Slx4 from other structure-specific nucleases, and allows them to resolve HR-dependent $\mathrm{HJs}$, but also analogues of replication forks, and presumably reversed fork intermediates, which are structurally equivalent to four-way $\mathrm{HJs}^{30,32,50}$. DNA intermediates that require detoxification by Yen1 arise in Dna2 helicase-defective cells in the absence of Rad52 (Fig. 6e), and in the presence of Mus81-Mms4, which we find in the active, hyperphosphorylated ${ }^{40}$ form in post-replicative dna2-2 cells (Supplementary Fig. 8). Therefore, Yen1 appears not to be primarily required to remove HR intermediates in dna2-2 cells, but instead for removing persistent replication intermediates, such as arrested forks or converged forks that fail to fuse (Fig. 6f,g). Of note, a similar activity of MUS81-EME1 towards late replication intermediates has been shown to avoid sister chromatid non-disjunction in human cells ${ }^{33,34}$. Thus, Yen 1 and other $\mathrm{HJ}$ resolving enzymes might function in complementary fashion, rather than redundantly, in targeting dead-end replication intermediates to protect chromosome segregation.

Persistent replication intermediates could explain chromosome non-disjunction in Dna2 helicase-defective cells (Fig. 5e), and it is tempting to speculate that the Dna2 helicase activity may be involved in replication fork remodelling reactions that facilitate fork recovery. This would be conceptually similar to the role of the Dna2 nuclease in replication restart at reversed forks through degradation of the regressed DNA branch ${ }^{21-23}$. A particularly attractive possibility is that fork reversal might occur and/or persist as a consequence of Dna2 helicase dysfunction. The resulting chicken-foot structure, which effectively contains a single-ended DNA double-strand break at the tip of the regressed DNA branch, could account for DNA damage checkpoint activation ${ }^{51}$, which we observe in Dna2 helicase-defective cells (Figs 2d,4a and 5c). A four-way chicken-foot DNA intermediate would also be amenable to resolution by Yen1; perhaps more so than by Mus81-Mms4, which is greatly stimulated by pre-existing nicks within DNA junctions, such as those that may be present in HR-dependent joint molecules and maturing $\mathrm{HJs}$ prior to a final ligation step $^{29,30,43}$. Importantly, and regardless of their exact structural features, the intermediates resolved by Yen1 in Dna2 helicase-defective cells constitute a first DNA target that is uniquely processed by Yen 1 . This demonstrates greater complexity in the uses of $\mathrm{HJ}$ resolvases in cells, and could explain the evolutionary conservation of Yen1/GEN1.

Intriguingly, Dna2 and Yen1 are both subject to CDK1-regulated nucleocytoplasmic shuttling ${ }^{52}$ (Fig. 7b). During $S$ phase, phosphorylation of Yen1 mediates nuclear exclusion, whereas phospho-Dna2 accumulates inside the nucleus. Thus, Dna2 can access sites of impaired DNA replication, and, consistently, has been found to form discrete nuclear foci during HU-induced replication arrest ${ }^{53}$. Dna2 helicase dysfunction gives rise to lesions that require Yen1 for resolution, but also triggers G2/M checkpoint activation, precluding dephosphorylation-dependent Yen1 activation and translocation to the nucleus on anaphase entry (Fig. 5a). Paradoxical though it may seem, this likely reflects a trade-off between the need to protect chromosomes from the DNA debranching activities of Yen1 during $S$ phase, while exploiting its unique biochemical properties to remove persistent chromosomal DNA links in $M$ phase. Indeed, Yen $1^{\text {on }}$ expression in $G 2 / M$ allows resolution of toxic DNA intermediates in dna2-2 cells (Supplementary Fig. 6), but constitutive expression is associated with MMS sensitivity, and tight control over the activities of $\mathrm{HJ}$ resolvases has been shown to limit sister chromatid exchange and the risk of loss of heterozygosity ${ }^{40,54-56}$. Despite the risk of terminal G2/M arrest, Yen1 effectively maintains the viability of Dna2 helicase-defective cells in unperturbed conditions (Fig. 2a), indicating its late activation as an elegant failsafe mechanism that allows Yen1 to act indiscriminately on DNA structures that resemble normal replication intermediates, identified as aberrant only by their presence at the wrong time in the cell cycle. In future, it will be interesting to see whether Yen1 represents a more general surveillance nuclease for aberrant replication intermediates that persist into anaphase. Strong G2/M checkpoint signalling and terminal arrest at the G2/M boundary might have precluded the detection of Yen1 functions downstream of repair and replication factors other than DNA2 in large-scale screening efforts thus far.

Loss and overexpression of DNA2 has been observed in human cancers and cancer cell lines ${ }^{12,57,58}$, while haploinsufficiency promotes cancer formation in heterozygous DNA2-knockout mice ${ }^{9}$. This suggests a complex role in cancer, where genome instability caused by impaired DNA2 function may drive tumorigenesis, whereas upregulation of DNA2 may help cancer cells to survive continuous DNA replication stress. Interestingly, a homozygous mutation in DNA2 has recently been identified in patients with Seckel syndrome ${ }^{59}$, a disease associated with a compromised response to replication fork stalling on the cellular level ${ }^{60}$. Depletion of DNA2 in mammalian cells recapitulates many of the phenotypes seen in Dna2-defective yeast, including sensitivity to replication stress, elevated DNA damage, chromosome instability and G2/M cell cycle delay ${ }^{9-12}$, indicating functional conservation. Our results implicate the elusive Dna2 helicase in replication fork recovery. Yen 1 provides a downstream survival pathway, along which toxic DNA intermediates that arise when the Dna2 helicase activity fails to respond adequately to replication fork stalling are resolved. Similar two-tiered mechanisms may contribute to the aetiology of human pathologies involving DNA2. 

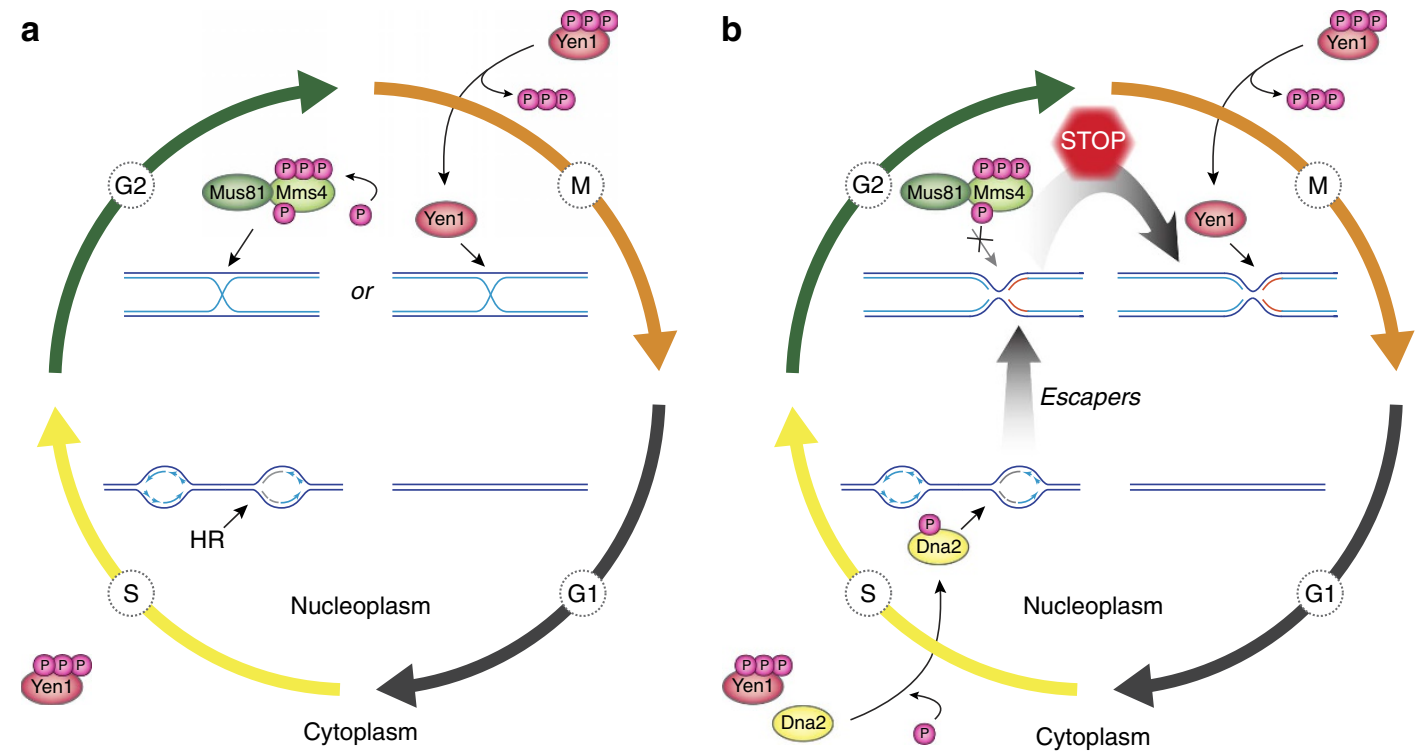

Figure 7 | Model for a two-tiered response to replication stress by Dna2 and Yen1. (a) Canonical role of Yen1 in the resolution of HR intermediates that arise during HR-mediated recovery of stalled replication forks. Mus81-Mms4 is activated by hyperphosphorylation in G2/M, prior to activation and nuclear import of Yen1 upon anaphase onset. Thus, HR intermediates are predominantly cleaved by Mus81-Mms4, with Yen1 acting as a catchall in M phase to remove persistent recombination structures in time for chromosome segregation. (b) Parallel to HR, the Dna2 helicase is tending to stalled replication forks. Replication intermediates that escape the attention of Dna2 give rise to toxic structures that are sensed by the DNA damage checkpoint, but which are refractory to processing by Mus81-Mms4. At anaphase entry, Yen1 is activated and uniquely resolves persistent replication intermediates, averting mitotic catastrophe. See text for details.

\section{Methods}

Recombinant proteins. Wild-type Dna2, Dna2 ${ }^{\mathrm{R} 1253 \mathrm{Q}}, \mathrm{Dna}{ }^{\mathrm{E} 675 \mathrm{~A}}$ and $\mathrm{Dna} 2^{\mathrm{K} 1080 \mathrm{E}}$ were expressed from a modified pGAL:DNA2 vector, adding N-terminal FLAG and $\mathrm{HA}$ tags and a C-terminal $6 \times$ His tag, and purified as described previously ${ }^{36,37}$. RPA protein was expressed and purified as described ${ }^{61}$.

Nuclease/helicase and ATPase assays. Experiments were carried out and analysed as described ${ }^{36,37}$. In brief, we used an assay that couples ATP hydrolysis to oxidation of NADH to determine the rate of ATP hydrolysis by Dna2 variants by following the decrease in optical absorbance by NADH at $340 \mathrm{~nm}$ over time. Kinetic plots of ATP hydrolysis were derived by calculating the amount of ATP hydrolysed per time interval. Fifteen microlitres reactions contained $25 \mathrm{mM}$ Tris-acetate ( $\mathrm{pH}$ 7.5), $2 \mathrm{mM}$ magnesium acetate, $1 \mathrm{mM}$ ATP, $1 \mathrm{mM}$ dithiothreitol, $0.1 \mathrm{mg} \mathrm{ml}^{-1}$ bovine serum albumin, $1 \mathrm{mM}$ phosphoenolpyruvate, $16 \mathrm{U} \mathrm{ml}^{-1}$ pyruvate kinase, $1 \mathrm{nM}$ DNA substrate, $16.8 \mathrm{nM}$ RPA and Dna2 proteins as indicated. Nuclease assays were incubated at $30^{\circ} \mathrm{C}$ for $30 \mathrm{~min}$. For analysis by denaturing polyacrylamide electrophoresis, samples were heat-denatured in formamide. DNA substrates were assembled using oligonucleotides X12-3 and X12-4SC, and PC 92 and X12-4SC for the 19 and 30 nt $5^{\prime}$-tailed DNA substrates, respectively ${ }^{36,37}$; the $5^{\prime}$-flapped DNA substrate consisted of oligonucleotides X12-4NC, Flap 19 X12-4C, and 292, as described ${ }^{37}$. Where indicated, oligonucleotides were ${ }^{32} \mathrm{P}$-labelled at the $5^{\prime}$-end using $\left[\gamma^{-}{ }^{32} \mathrm{P}\right]$ ATP and T4 polynucleotide kinase (New England Biolabs). Unincorporated nucleotides were removed using MicroSpin G25 columns (GE Healthcare) before annealing the respective DNA substrates.

Yeast strains and plasmids. S. cerevisiae strains (Supplementary Table 1) were derived from BY4741 (ref. 62) using standard methods. The dna2-2 allele was generated using pop-in/pop-out mutagenesis ${ }^{63}$, and the DNA damage sensitivity of the resulting strain could be complemented with plasmid-borne wild-type DNA2 cloned into vector pAG416GPD-ccdB (pDNA2) (Supplementary Fig. 2b). For constitutive expression, YEN1 was cloned into vector pAG416GPD-ccdB or pAG416GPD-ccdB-EGFP ${ }^{64}$, and site-directed mutagenesis was performed to generate a catalytically inactive form of Yen1 bearing the mutations E193A and E195A. Yellow fluorescent protein (YFP)-tagged Rad52 was expressed from its endogenous promoter using centromeric plasmid pWJ1213 (ref. 65). If not stated otherwise, all strains were cultured at $30^{\circ} \mathrm{C}$ using YPAD media. YEN1 ${ }^{\text {on }}$ was cloned into vector pAG416GPD-ccdB, or pYES-DEST52 (Invitrogen) for expression from a GAL1 promoter in YPLG medium with $2 \%(\mathrm{w} / \mathrm{v})$ galactose and $1 \%(\mathrm{w} / \mathrm{v})$ raffinose. Antibodies used to monitor the expression of tagged proteins were Abcam mouse monoclonal anti-V5 antibody ab27671, and Sigma mouse monoclonal anti-Myc antibody 9E10. Santa Cruz Biotechnology goat polyclonal
anti-Mcm2 antibody yN-19 was routinely used to ensure gel lanes were equally loaded for total protein.

Cell viability and drug sensitivity assays. Doubling times were determined as described ${ }^{66}$ and averaged over at least three independent experiments. For microscopic determination of cell cycle stage (budding index), an average of 400 cells per strain and replicate were scored. Plating efficiency as a measure of strain viability was determined by colony outgrowth after plating a defined number of cells. The number of colonies formed after 3-4 days at $30^{\circ} \mathrm{C}$ was divided by the number of cells plated as quantified in haemocytometer counts. For drop assays, exponentially growing cells were normalized to $10^{7}$ cells $\mathrm{ml}^{-1}$, and $2 \mu \mathrm{l}$ drops of tenfold serial dilutions were spotted onto the appropriate medium with or without MMS, HU or CPT. If not stated otherwise, plates were incubated for $3-4$ days at $30^{\circ} \mathrm{C}$. For liquid survival assays, overnight cultures were diluted to $\mathrm{OD}_{600}=0.1-0.2$ and grown for $4 \mathrm{~h}$, then synchronized with $\alpha$-factor in G1 and released into YPAD containing $50 \mathrm{mM} \mathrm{HU}$ for $120 \mathrm{~min}$. Relevant dilutions were plated onto YPAD plates and colonies were counted after 3-4 days.

Mitotic time-courses. For time-course experiments, cells were grown exponentially $\left(\mathrm{OD}_{600}=0.4-0.6\right)$ and synchronized by addition of $\alpha$-factor (routinely $>95 \%$ unbudded cells for wild-type, $\geq 90 \%$ for Dna2 helicase-defective strains). Cells were then harvested, washed and released into YPAD containing $50 \mathrm{mM} \mathrm{HU}$ for $2 \mathrm{~h}$. After HU wash-out, cells were cultured in drug-free medium. Aliquots for flow cytometry, Western blot analysis and microscopy were withdrawn at regular intervals. Where indicated, $\alpha$-factor or nocodazole $\left(15 \mu \mathrm{g} \mathrm{ml}^{-1}\right)$ was added during and/or after treatment.

Analysis of Rad53-phosphorylation. TCA-precipitated proteins were separated by SDS-PAGE using precast gels (Invitrogen), and blotted onto polyvinylidene difluoride membranes using a Bio-Rad Turbo blot system. Rad53 protein was detected using a custom-made mouse monoclonal antibody ${ }^{67}$. Uncropped immunoblots are shown in Supplementary Fig. 9.

Flow cytometry. Cells were fixed overnight in $70 \%$ ethanol at $4{ }^{\circ} \mathrm{C}$ with rotation and processed as described ${ }^{68}$. Cells were then washed and resuspended using $50 \mathrm{mM} \mathrm{Na}$-citrate ( $\mathrm{pH}$ 7). After brief sonication, RNase A was added $\left(0.25 \mathrm{mg} \mathrm{ml}^{-1}\right)$, and cells were incubated overnight at $37^{\circ} \mathrm{C}$, washed, and resuspended in $50 \mathrm{mM} \mathrm{Na}$-citrate ( $\mathrm{pH}$ 7) containing $16 \mu \mathrm{g} \mathrm{ml}^{-1}$ propidium iodide. Measurements of DNA content were done using a BD LSR II flow cytometer 
(Becton Dickinson) operated with BD FACSDiva software. Data was processed with FlowJo (TreeStar).

Microscopy. DIC images were obtained using a Zeiss Axio Imager Z1 with a PlanApochromat $63 \times / 1.4$ DIC oil objective (Zeiss) and an AxioCam camera controlled by ZEN Blue 2012 software. To analyse nuclear DNA and chromosome segregation, cells were fixed with $70 \%$ ethanol for $5 \mathrm{~min}$ at room temperature and stained with 4,6-diamidino-2-phenylindole (DAPI) $\left(50 \mathrm{ng} \mathrm{ml}^{-1}\right)$. For Yen1-EGFP and Rad52-YFP analyses, cells were fixed in $4 \%$ paraformaldehyde for $3 \mathrm{~min}$ at room temperature and stained with DAPI. Confocal images were collected using a Zeiss Axio Imager M1/Yokogawa CSU-X1 scanhead multipoint confocal microscope with a Plan-Neofluar $100 \times / 1.45$ oil objective and EM-CCD Cascade II camera (Photometrics) controlled by Metamorph 7.7.2 software (Molecular Devices), or a Rolera Thunder Back Illuminated EM-CCD camera (Q Imaging) controlled by VisiView software (Visitron Systems). Stacks of $>20$ optical slices separated by $200 \mathrm{~nm}$ were collected, and images of two-dimensional projections were prepared with ImageJ software (Fiji).

Analysis of rDNA replication by 2D gel electrophoresis. Synchronized or exponentially growing cells were harvested by centrifugation, and genomic DNA was purified using G-20 columns (Qiagen) before digestion with BglII. For S phase samples, aliquots were withdrawn every $10 \mathrm{~min}$ for $60 \mathrm{~min}$ on release from $\alpha$-factorinduced G1 arrest, and pooled before preparing genomic DNA. Ethanol-purified DNA digests $(2.5 \mu \mathrm{g})$ were subjected to $2 \mathrm{D}$ gel analysis as described ${ }^{69}$, with minor modifications. The first dimension gel $\left(0.4 \%\right.$ agarose in TBE) was run at $1 \mathrm{~V} \mathrm{~cm}^{-1}$ at room temperature for $16 \mathrm{~h}$. The second dimension gel $(1.5 \%$ in TBE containing $0.3 \mathrm{~g} \mathrm{ml}^{-1}$ ethidium bromide) was run at $5 \mathrm{~V} \mathrm{~cm}^{-1}$ at $4{ }^{\circ} \mathrm{C}$ in circulating TBE buffer for $5 \mathrm{~h}$. The DNA was then blotted onto Hybond XL membrane (Amersham) by capillary transfer in $0.4 \mathrm{~N} \mathrm{NaOH}$. After UV-crosslinking, the membrane was blocked with ssDNA, probed for rDNA, and washed according to instructions by the manufacturer. A DNA template for the Southern probe was prepared by PCR from genomic DNA using primers 5'-GCCATTTACAAAAACATAACG and $5^{\prime}$-GGGC CTAGTTTAGAGAGAAGT ${ }^{49}$. The radiolabelled probe was then synthesized in the presence of $\left[\alpha-{ }^{32} \mathrm{P}\right]$ dCTP and $\left[\alpha^{-32} \mathrm{P}\right]$ dATP using Klenow fragment polymerization (New England Biolabs/Bioconcept). Radioactive Southern blots were imaged using a phosphorimager screen (Kodak) and a Typhoon ${ }^{\mathrm{TM}} 9400$ system (GE Healthcare), and quantified using ImageQuant TL v2005 software as described ${ }^{70}$. In brief, the signal intensities for individual image objects were normalized to the intensity of the $1 \mathrm{~N}$ spot after background correction. The fold change was calculated by dividing the normalized signal intensity of each intermediate in G2 phase by the corresponding signal in S phase. For the experiment with Yen $1^{\text {on }}$ expression in dna2-2 cells, the normalized signal intensity for each scrutinized DNA intermediate in the strain harbouring the Yen $1^{\text {on }}$ construct was divided by the corresponding signal in the strain with empty vector.

Data availability. The authors declare that all data supporting the findings of this study are available within the article and its Supplementary Information, or from the corresponding author on request.

\section{References}

1. Torres-Rosell, J. et al. Anaphase onset before complete DNA replication with intact checkpoint responses. Science 315, 1411-1415 (2007).

2. Aguilera, A. \& Gómez-González, B. Genome instability: a mechanistic view of its causes and consequences. Nat. Rev. Genet. 9, 204-217 (2008).

3. Halazonetis, T. D., Gorgoulis, V. G. \& Bartek, J. An oncogene-induced DNA damage model for cancer development. Science 319, 1352-1355 (2008).

4. Hustedt, N., Gasser, S. \& Shimada, K. Replication checkpoint: tuning and coordination of replication forks in S phase. Genes 4, 388-434 (2013).

5. Lambert, S., Froget, B. \& Carr, A. M. Arrested replication fork processing: interplay between checkpoints and recombination. DNA Repair (Amst.) 6, 1042-1061 (2007).

6. Atkinson, J. \& McGlynn, P. Replication fork reversal and the maintenance of genome stability. Nucleic Acids Res. 37, 3475-3492 (2009).

7. Petermann, E. \& Helleday, T. Pathways of mammalian replication fork restart. Nat. Rev. Mol. Cell Biol. 11, 683-687 (2010).

8. Budd, M. E. \& Campbell, J. L. A yeast gene required for DNA replication encodes a protein with homology to DNA helicases. Proc. Natl Acad. Sci. USA 92, 7642-7646 (1995).

9. Lin, W. et al. Mammalian DNA2 helicase/nuclease cleaves G-quadruplex DNA and is required for telomere integrity. EMBO J. 32, 1425-1439 (2013).

10. Duxin, J. P. et al. Okazaki fragment processing-independent role for human Dna2 enzyme during DNA replication. J. Biol. Chem. 287, 21980-21991 (2012).

11. Duxin, J. P. et al. Human Dna2 is a nuclear and mitochondrial DNA maintenance protein. Mol. Cell Biol. 29, 4274-4282 (2009).
12. Peng, G. et al. Human nuclease/helicase DNA2 alleviates replication stress by promoting DNA end resection. Cancer Res. 72, 2802-2813 (2012).

13. Cheng, E. et al. Genome rearrangements caused by depletion of essential DNA replication proteins in Saccharomyces cerevisiae. Genetics 192, 147-160 (2012).

14. Formosa, T. \& Nittis, T. Dna2 mutants reveal interactions with Dna polymerase alpha and Ctf4, a Pol alpha accessory factor, and show that full Dna2 helicase activity is not essential for growth. Genetics 151, 1459-1470 (1999).

15. Aravind, L., Walker, D. R. \& Koonin, E. V. Conserved domains in DNA repair proteins and evolution of repair systems. Nucleic Acids Res. 27, 1223-1242 (1999).

16. Bae, S. H. et al. Dna2 of Saccharomyces cerevisiae possesses a single-stranded DNA-specific endonuclease activity that is able to act on double-stranded DNA in the presence of ATP. J. Biol. Chem. 273, 26880-26890 (1998).

17. Kumar, S. \& Burgers, P. M. Lagging strand maturation factor Dna2 is a component of the replication checkpoint initiation machinery. Genes Dev. 27, 313-321 (2013).

18. Bae, S. H., Bae, K. H., Kim, J. A. \& Seo, Y. S. RPA governs endonuclease switching during processing of Okazaki fragments in eukaryotes. Nature 412, 456-461 (2001).

19. Bae, S. H. \& Seo, Y. S. Characterization of the enzymatic properties of the yeast Dna2 helicase/endonuclease suggests a new model for Okazaki fragment processing. J. Biol. Chem. 275, 38022-38031 (2000).

20. Cejka, P. DNA end resection: nucleases team up with the right partners to initiate homologous recombination. J. Biol. Chem. 19, 169-175 (2014).

21. Hu, J. et al. The intra-S phase checkpoint targets Dna2 to prevent stalled replication forks from reversing. Cell 149, 1221-1232 (2012).

22. Lai, M. S. \& Foiani, M. Dna2 offers support for stalled forks. Cell 149, 1181-1183 (2012).

23. Thangavel, S. et al. DNA2 drives processing and restart of reversed replication forks in human cells. J. Cell Biol. 208, 545-562 (2015).

24. Karanja, K. K., Lee, E. H., Hendrickson, E. A. \& Campbell, J. L. Preventing over-resection by DNA2 helicase/nuclease suppresses repair defects in Fanconi anemia cells. Cell Cycle 13, 1540-1550 (2014).

25. Higgs, M. R. et al. BOD1L is required to suppress deleterious resection of stressed replication forks. Mol. Cell 59, 462-477 (2015).

26. Araki, Y., Kawasaki, Y., Sasanuma, H., Tye, B. K. \& Sugino, A. Budding yeast mom10/dna43 mutant requires a novel repair pathway for viability. Genes Cells 8, 465-480 (2003).

27. Lee, K. H. et al. The endonuclease activity of the yeast Dna2 enzyme is essential in vivo. Nucleic Acids Res. 28, 2873-2881 (2000).

28. Budd, M. E. et al. A network of multi-tasking proteins at the DNA replication fork preserves genome stability. PLoS Genet. 1, e61 (2005).

29. Ip, S. C. Y. et al. Identification of Holliday junction resolvases from humans and yeast. Nature 456, 357-361 (2008).

30. Schwartz, E. K. \& Heyer, W.-D. Processing of joint molecule intermediates by structure-selective endonucleases during homologous recombination in eukaryotes. Chromosoma 120, 109-127 (2011).

31. Sarbajna, S. \& West, S. C. Holliday junction processing enzymes as guardians of genome stability. Trends Biochem. Sci. 39, 409-419 (2014).

32. Rass, U. Resolving branched DNA intermediates with structure-specific nucleases during replication in eukaryotes. Chromosoma 122, 499-515 (2013).

33. Naim, V., Wilhelm, T., Debatisse, M. \& Rosselli, F. ERCC1 and MUS81-EME1 promote sister chromatid separation by processing late replication intermediates at common fragile sites during mitosis. Nat. Cell Biol. 15, 1008-1015 (2013).

34. Ying, S. et al. MUS81 promotes common fragile site expression. Nat. Cell Biol. 15, 1001-1007 (2013).

35. Budd, M. E., Choe, W.-C. \& Campbell, J. L. The nuclease activity of the yeast Dna2 protein, which is related to the RecB-like nucleases, is essential in vivo. J. Biol. Chem. 275, 16518-16529 (2000).

36. Levikova, M., Klaue, D., Seidel, R. \& Cejka, P. Nuclease activity of Saccharomyces cerevisiae Dna2 inhibits its potent DNA helicase activity. Proc. Natl Acad. Sci. USA 110, E1992-E2001 (2013).

37. Levikova, M. \& Cejka, P. The Saccharomyces cerevisiae Dna2 can function as a sole nuclease in the processing of Okazaki fragments in DNA replication. Nucleic Acids Res. 43, 7888-7897 (2015).

38. Bae, S. H. et al. Coupling of DNA helicase and endonuclease activities of yeast Dna2 facilitates Okazaki fragment processing. J. Biol. Chem. 277, 26632-26641 (2002).

39. Blanco, M. G., Matos, J., Rass, U., Ip, S. C. Y. \& West, S. C. Functional overlap between the structure-specific nucleases Yen1 and Mus81-Mms4 for DNA-damage repair in S. cerevisiae. DNA Repair (Amst.) 9, 394-402 (2010).

40. West, S. C. et al. Resolution of recombination intermediates: mechanisms and regulation. Cold Spring Harb. Symp. Quant. Biol. 80, 103-109 (2015). 
41. Fiorentino, D. F. \& Crabtree, G. R. Characterization of Saccharomyces cerevisiae dna2 mutants suggests a role for the helicase late in $\mathrm{S}$ phase. Mol. Biol. Cell 8, 2519-2537 (1997).

42. Budd, M. E., Antoshechkin, I. A., Reis, C., Wold, B. J. \& Campbell, J. L. Inviability of a DNA2 deletion mutant is due to the DNA damage checkpoint. Cell Cycle 10, 1690-1698 (2011).

43. Blanco, M. G., Matos, J. \& West, S. C. Dual control of Yen1 nuclease activity and cellular localization by $\mathrm{Cdk}$ and $\mathrm{Cdc} 14$ prevents genome instability. Mol. Cell 54, 94-106 (2014).

44. Budd, M. E. \& Campbell, J. L. The pattern of sensitivity of yeast dna2 mutants to DNA damaging agents suggests a role in DSB and postreplication repair pathways. Mutat. Res. 459, 173-186 (2000).

45. Lisby, M., Rothstein, R. \& Mortensen, U. H. Rad52 forms DNA repair and recombination centers during S phase. Proc. Natl Acad. Sci. USA 98, 8276-8282 (2001).

46. Yimit, A., Riffle, M. \& Brown, G. W. Genetic regulation of Dna2 localization during the DNA damage response. G3 (Bethesda) 5, 1937-1944 (2015).

47. Ho, C. K., Mazón, G., Lam, A. F. \& Symington, L. S. Mus81 and Yen1 promote reciprocal exchange during mitotic recombination to maintain genome integrity in budding yeast. Mol. Cell 40, 988-1000 (2010).

48. Brewer, B. J. \& Fangman, W. L. A replication fork barrier at the $3^{\prime}$ end of yeast ribosomal RNA genes. Cell 55, 637-643 (1988).

49. Weitao, T., Budd, M., Hoopes, L. L. M. \& Campbell, J. L. Dna2 helicase/nuclease causes replicative fork stalling and double-strand breaks in the ribosomal DNA of Saccharomyces cerevisiae. J. Biol. Chem. 278, 22513-22522 (2003).

50. Neelsen, K. J. \& Lopes, M. Replication fork reversal in eukaryotes: from dead end to dynamic response. Nat. Rev. Mol. Cell Biol. 16, 207-220 (2015).

51. Fugger, K. et al. FBH1 catalyzes regression of stalled replication forks. Cell Rep. 10, 1749-1757 (2015).

52. Kosugi, S., Hasebe, M., Tomita, M. \& Yanagawa, H. Systematic identification of cell cycle-dependent yeast nucleocytoplasmic shuttling proteins by prediction of composite motifs. Proc. Natl Acad. Sci. USA 106, 10171-10176 (2009).

53. Tkach, J. M. et al. Dissecting DNA damage response pathways by analysing protein localization and abundance changes during DNA replication stress. Nat. Cell Biol. 14, 966-976 (2012).

54. Szakal, B. \& Branzei, D. Premature Cdk1/Cdc5/Mus81 pathway activation induces aberrant replication and deleterious crossover. EMBO J. 32, 1155-1167 (2013).

55. Matos, J., Blanco, M. G. \& West, S. C. Cell-cycle kinases coordinate the resolution of recombination intermediates with chromosome segregation. Cell Rep. 4, 76-86 (2013).

56. Chan, Y. W. \& West, S. C. Spatial control of the GEN1 Holliday junction resolvase ensures genome stability. Nat. Commun. 5, 4844 (2014).

57. Lee, S. H., Kim, Y. R., Yoo, N. J. \& Lee, S. H. Mutation and expression of DNA2 gene in gastric and colorectal carcinomas. Korean J. Pathol. 44, 354-349 (2010).

58. Strauss, C. et al. The DNA2 nuclease/helicase is an estrogen-dependent gene mutated in breast and ovarian cancers. Oncotarget 5, 9396-9409 (2014).

59. Shaheen, R. et al. Genomic analysis of primordial dwarfism reveals novel disease genes. Genome Res. 24, 291-299 (2014).

60. Alderton, G. K. et al. Seckel syndrome exhibits cellular features demonstrating defects in the ATR-signalling pathway. Hum. Mol. Genet. 13, 3127-3138 (2004).

61. Kantake, N., Sugiyama, T., Kolodner, R. D. \& Kowalczykowski, S. C. The recombination-deficient mutant RPA (rfal-t11) is displaced slowly from single-stranded DNA by Rad51 protein. J. Biol. Chem. 278, 23410-23417 (2003).

62. Brachmann, C. et al. Designer deletion strains derived from Saccharomyces cerevisiae S288C: a useful set of strains and plasmids for PCR-mediated gene disruption and other applications. Yeast 14, 115-132 (1998).

63. Scherer, S. \& Davis, R. W. Replacement of chromosome segments with altered DNA sequences constructed in vitro. Proc. Natl Acad. Sci. USA 76, 4951-4955 (1979).
64. Alberti, S., Gitler, A. D. \& Lindquist, S. A suite of Gateway cloning vectors for high-throughput genetic analysis in Saccharomyces cerevisiae. Yeast 24, 913-919 (2007).

65. Feng, Q. et al. Rad52 and Rad59 exhibit both overlapping and distinct functions. DNA Repair (Amst.) 6, 27-37 (2007).

66. Bernstein, K. A. et al. Sgs1 function in the repair of DNA replication intermediates is separable from its role in homologous recombinational repair. EMBO J. 28, 915-925 (2009).

67. Seeber, A., Dion, V. \& Gasser, S. M. Checkpoint kinases and the INO80 nucleosome remodeling complex enhance global chromatin mobility in response to DNA damage. Genes Dev. 27, 1999-2008 (2013).

68. Nash, G. B., Jones, J. G., Mikita, J. \& Dormandy, J. A. Methods and theory for analysis of flow of white cell subpopulations through micropore filters. Br. J. Haematol. 70, 165-170 (1988).

69. Huberman, J. A., Spotila, L. D., Nawotka, K. A., El-Assouli, S. M. \& Davis, L. R. The in vivo replication origin of the yeast 2 microns plasmid. Cell 51, 473-481 (1987).

70. Fumasoni, M., Zwicky, K., Vanoli, F., Lopes, M. \& Branzei, D. Error-free DNA damage tolerance and sister chromatid proximity during DNA replication rely on the Pol $\alpha /$ Primase/Ctf4 complex. Mol. Cell 57, 812-823 (2015).

\section{Acknowledgements}

We are indebted to S. Gasser, K. Shimada and A. Seeber for their generous technical help and reagents. We thank our colleagues S. Gasser, N. Thomä, and members of the Gasser and Rass laboratories for critical reading of the manuscript and helpful discussions. S. West and M. Blanco kindly provided the Yen $1^{\text {on }}$ construct. We are grateful for the assistance of H. Kohler from the Cell Sorting platform, and L. Gelman and S. Bourke from the Facility for Advanced Imaging and Microscopy at the Friedrich Miescher Institute. Work in the laboratory of P.C. is supported by Swiss National Science Foundation Professorship PP00P3 133636, and work in the laboratory of U.R. by the Swiss Cancer League \& Swiss Cancer Research and the Novartis Research Foundation.

\section{Author contributions}

G.Ö. and U.R. planned and analysed the experiments. G.Ö. performed the experiments with help from D.K. B.F. supported the two-dimensional gel analyses, and G.A.F. imaging and microscopy. M.L. and P.C. purified and analysed Dna2 in vitro. U.R. wrote the paper.

\section{Additional information}

Supplementary Information accompanies this paper at http://www.nature.com/ naturecommunications

Competing financial interests: The authors declare no competing financial interests

Reprints and permission information is available at http://npg.nature.com/ reprintsandpermissions/

How to cite this article: Ölmezer, G. et al. Replication intermediates that escape Dna2 activity are processed by Holliday junction resolvase Yen1. Nat. Commun. 7, 13157 doi: 10.1038/ncomms13157 (2016).

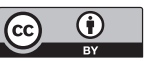

This work is licensed under a Creative Commons Attribution 4.0 International License. The images or other third party material in this article are included in the article's Creative Commons license, unless indicated otherwise in the credit line; if the material is not included under the Creative Commons license, users will need to obtain permission from the license holder to reproduce the material. To view a copy of this license, visit http://creativecommons.org/licenses/by/4.0/

(C) The Author(s) 2016 